\title{
Three-dimensional wedge filling in ordered and disordered systems
}

\author{
M. J. Greenall†§, A. O. Parry†, and J. M. \\ Romero-Enrique $\$$ \\ † Department of Mathematics, Imperial College London, 180 Queen's Gate, \\ London SW7 2BZ, United Kingdom \\ ¥ Departamento de Física Atómica, Molecular y Nuclear, Area de Física Teórica, \\ Universidad de Sevilla, Apartado de Correos 1065, 41080 Sevilla, Spain
}

\begin{abstract}
We investigate interfacial structural and fluctuation effects occurring at continuous filling transitions in $3 \mathrm{D}$ wedge geometries. We show that fluctuation-induced wedge covariance relations that have been reported recently for $2 \mathrm{D}$ filling and wetting have mean-field or classical analogues that apply to higher-dimensional systems. Classical wedge covariance emerges from analysis of filling in shallow wedges based on a simple interfacial Hamiltonian model and is supported by detailed numerical investigations of filling within a more microscopic Landau-like density functional theory. Evidence is presented that classical wedge covariance is also obeyed for filling in more acute wedges in the asymptotic critical regime. For sufficiently short-ranged forces mean-field predictions for the filling critical exponents and covariance are destroyed by pseudo-one-dimensional interfacial fluctuations. In this filling fluctuation regime we argue that the critical exponents describing the divergence of lengthscales are related to values of the interfacial wandering exponent $\zeta(d)$ defined for planar interfaces in (bulk) twodimensional $(d=2)$ and three-dimensional $(d=3)$ systems. For the interfacial height $l_{w} \sim(\theta-\alpha)^{-\beta_{w}}$, with $\theta$ the contact angle and $\alpha$ the wedge tilt angle, we find $\beta_{w}=\zeta(2) / 2(1-\zeta(3))$. For pure systems (thermal disorder) we recover the known result $\beta_{w}=1 / 4$ predicted by interfacial Hamiltonian studies whilst for random-bond disorder we predict the universal critical exponent $\beta \approx 0.59$ even in the presence of dispersion forces. We revisit the transfer matrix theory of three-dimensional filling based on an effective interfacial Hamiltonian model and discuss the interplay between breather, tilt and torsional interfacial fluctuations. We show that the coupling of the modes allows the problem to be mapped onto a quantum mechanical problem as conjectured by previous authors. The form of the interfacial height probability distribution function predicted by the transfer matrix approach is shown to be consistent with scaling and thermodynamic requirements for distances close to and far from the wedge bottom respectively.
\end{abstract}

PACS numbers: 68.08.Bc, 05.70.Np, 68.35.Md, 68.35.Rh

$\S$ Present address: School of Physics, The University of Edinburgh, Mayfield Road, Edinburgh EH9 3JZ, United Kingdom 


\section{Introduction}

Fluids adsorbed at micropatterned and sculpted solid surfaces may exhibit novel phase transitions and fluctuation effects compared to wetting behaviour at planar, heterogeneous walls 1, 2, 3. A striking example of the influence of substrate geometry on fluid adsorption is provided by a simple wedge geometry [5, 6, 7, 8, 9, 10, 11, 12. At two phase coexistence, a wedge-vapour interface is completely filled with liquid provided the contact angle $\theta$ is less than the wedge tilt angle $\alpha$. The phase transition from microscopic to macroscopic adsorption as $\theta \rightarrow \alpha^{+}$is referred to as filling and may be first-order or continuous. The conditions for continuous wedge (and also conic) filling are less restrictive than for continuous (critical) wetting at planar walls [8, 9] and give some hope that large-scale fluctuation effects associated with interfacial unbinding may be observable in the laboratory. Recent effective interfacial Hamiltonian studies have shown that fluctuation effects at continuous filling transitions exhibit a number of intriguing features. For three-dimensional wedge filling transitions critical singularities are believed to be far stronger than those characteristic of critical wetting transitions reflecting the anisotropy of soft-mode interfacial fluctuations induced by the wedge geometry. In particular for pure systems with sufficiently short-ranged forces the mean interfacial height $l_{w}$ (measured above the wedge bottom) and roughness $\xi_{\perp}$ are comparable and diverge with the same universal critical exponent $\beta_{w}=\nu_{\perp}=1 / 4$ [8, 9]. These predictions are in very good agreement with Monte Carlo simulation studies of filling within more microscopic Ising and lattice polymer models [13, 14. For two-dimensional wedge filling on the other hand fluctuation effects are interesting for a different reason. Studies of fluctuation-dominated filling in both ordered (pure) [10] and disordered (random-bond) [1] systems show that some observables, such as the mid-point height probability distribution function, show scaling properties which are identical with short-ranged critical wetting transitions. The only influence of the wedge geometry is to shift the effective value of the contact angle from $\theta$ to $\theta-\alpha$ - a feature which has been referred to as wedge covariance [12. This "hidden symmetry" between wetting and filling appears to restrict the allowed values of the critical exponents at both 2D filling and wetting and leads to new some insights into the properties of critical wetting transitions.

The present paper focuses on the structural and fluctuation properties of 3D wedge filling transitions. We begin with a discussion of wedge covariance and illustrate using a simple interfacial model of filling in shallow wedges that the fluctuation-induced covariance observed for 2D systems has a mean-field or classical precursor for wetting and filling in systems with short-ranged forces. Thus if $l_{\pi}(\theta)$ denotes the mean-field result for the contact angle dependence of the critical wetting layer thickness, then in the wedge geometry the corresponding mean-field result for the mid-point height at bulk coexistence is

$$
l_{w}(\theta, \alpha)=l_{\pi}(\theta-\alpha)
$$

The predictions of the interfacial model are supported by a detailed numerical study of filling in a Landau-like density functional model that indicate this classical covariance is obeyed for both shallow and more acute wedges. The implications of classical wedge covariance for the structure of interfacial models of short-ranged filling and wetting are discussed. In the second part of our paper we turn our attention to fluctuation effects at 3D wedge filling and present a general scaling argument that relates the values of the critical exponents for $3 \mathrm{D}$ wedge filling to the wandering exponent of 
planar, two-dimensional and three-dimensional fluid interfaces. Thus, for example the critical exponent $\beta_{w}$ is identified as

$$
\beta_{w}=\frac{\zeta(2)}{2(1-\zeta(3))}
$$

where $\zeta(d)$ is the standard wandering exponent for a planar-like interface in a $d$ dimensional bulk system. Our expressions recover the values quoted above for pure systems, corresponding to thermal fluctuations, and also allow us to discuss 3D filling in disordered systems. We also discuss the cross-over from three-dimensional to twodimensional wedge filling as one lowers the number of dimensions of translational invariance along the wedge. The change in the nature of fluctuation effects and critical singularities in this wedge compactification process highlights a remarkable numerical coincidence concerning the value of the wandering exponent for planar interfaces at the lower marginal dimension for wedge filling. In the final part of our study we focus on 3D filling in pure systems and address some problems that have been highlighted concerning the transfer-matrix analysis of a pseudo-one-dimensional effective Hamiltonian model. We argue that problems associated with the choice of the appropriate measure in the functional integral can be avoided if, in addition to breather-mode excitations of the interface, we also allow for tilt and torsional degrees of freedom. This more accurate formulation of the theory allows us to determine the universal scaling function associated with the density profile in the wedge. The form of this function is shown to have the correct short-distance and large-distance behaviour dictated by scaling theory and macroscopics respectively.

\section{Background theory}

\subsection{Thermodynamics and critical exponents}

Consider a 3D wedge formed from the intersection of two smooth, planar walls that meet at angles $\alpha$ to the $z=0$ plane forming a wedge with opening angle $\pi-2 \alpha$. The parallel displacement vector in the $z=0$ plane is written $\mathbf{x}=(x, y)$ with cartesians $(x, y)$ measuring distances across and along the wedge respectively. Thus the height of the wall above the $z=0$ plane is described by a height function $z_{w}(\mathbf{x})=\tan \alpha|x|$. We suppose that the wedge is in contact with a three-dimensional bulk vapour at subcritical temperature $T$ and chemical potential $\mu$ tuned to bulk two-phase coexistence $\mu=\mu_{\text {sat }}(T)^{-}$. Provided the contact angle $\theta(T)$ of the sessile drop (defined for the planar wall-fluid interface) is less than ninety degrees the wedge preferentially adsorbs a volume of liquid near its bottom. Partial and complete filling refer to situations where the adsorption is microscopic and macroscopic respectively. The separatrix between partial and complete filling follows from simple thermodynamic arguments first discussed by Concus and Finn [4] some thirty years ago (see also Pomeau [5] and Hauge [6]). Let $V$ denote the accessible volume of the fluid and $A, L$ the area and length of the wedge respectively. The total grand potential $\Omega$ contains the macroscopic contributions

$$
\Omega=-p V+\sigma_{w v} A+f_{w} L
$$

where $\sigma_{w v}$ is the surface tension of the wall-vapour interface and $f_{w}$ is the excess wedge free energy. Now suppose we are at bulk coexistence and imagine that the wedge is filled to a height $l_{w}$ (see Figure 11). Macroscopically the liquid-vapour interface must 


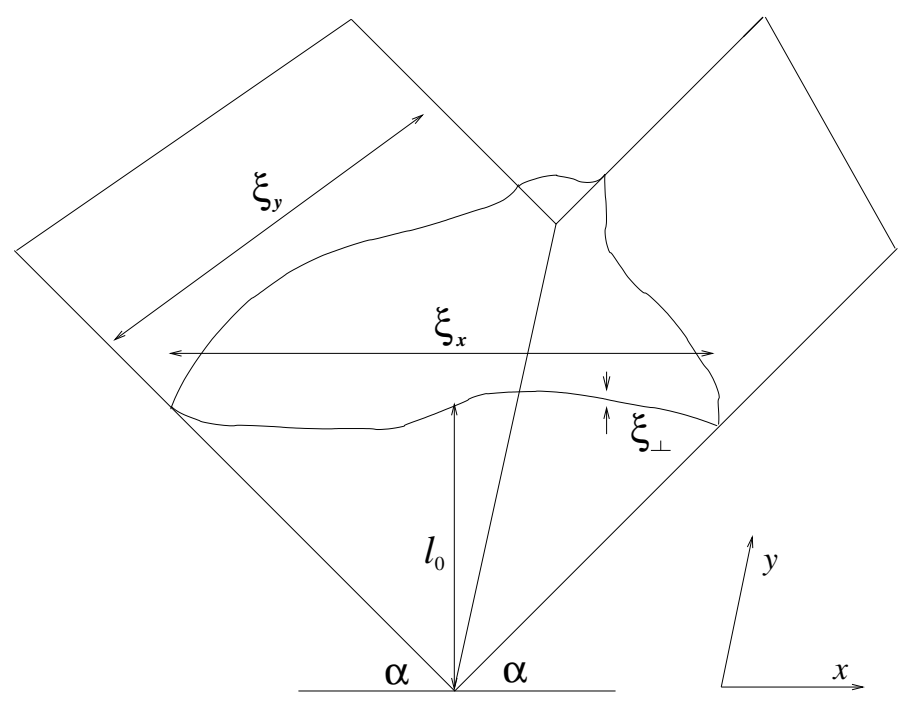

Figure 1. Schematic illustration of a typical interfacial configuration in the 3D wedge geometry and the typical diverging lengthscales at the filling transition. Note that $l_{w}=\left\langle l_{0}\right\rangle$.

be flat and hence $f_{w}$ must contain a thermodynamic contribution

$$
f_{w}=\frac{2 \sigma_{l v}(\cos \alpha-\cos \theta) l_{w}}{\sin \alpha}
$$

where $\sigma_{l v}$ is the liquid-vapour tension and we have used Young's equation. It follows that for $\theta<\alpha$ the free energy can be lowered by completely filling the wedge. For $\theta>\alpha$ on the other hand the equilibrum value of $l_{w}$ must be finite and arises from a balance between thermodynamics and internal energy (intermolecular forces) or entropic (fluctuation) contributions. Thus by either increasing the tilt angle at constant $T$ or increasing the temperature at constant angle (assuming the usual scenario where the contact angle decreases with temperature) one can induce a transition from partial to complete filling at a temperature $T_{\text {fill }}$ satisfying

$$
\theta\left(T_{\text {fill }}\right)=\alpha
$$

Filling transitions may be either first- or second-order corresponding to the discontinuous or continuous divergence of the mean interfacial height $l_{w}$ respectively. Importantly, the conditions for continuous filling are less restrictive than for continuous wetting transitions and wedges made from walls that exhibit first-order wetting (at some higher temperature $T_{w e t}$ ) may still exhibit continuous filling [8, 9]. The most important diverging lengthscales which characterise the the transition are the mean mid-point height $l_{w}$, the perpendicular correlation length $\xi_{\perp}$ at the mid-point defined from the root-mean-square variance, and the correlation length $\xi_{y}$ describing fluctuations along the wedge. Correlations across the wedge are described by a lengthscale which is trivially related to the mean-height: $\xi_{x} \sim l_{w} \cot \alpha$. At bulk coexistence critical exponents for the divergence of these length scales are identified according to

$$
l_{w} \sim t^{-\beta_{w}} \quad, \quad \xi_{\perp} \sim t^{-\nu_{\perp}} \quad, \quad \xi_{y} \sim t^{-\nu_{y}}
$$


where the temperature-like scaling field $t \propto\left(T_{\text {fill }}-T\right) \propto(\theta-\alpha)$. The phenomenology is very similar for the case of $2 \mathrm{D}$ wedge filling except there is no analogue of the correlation length $\xi_{y}$.

\subsection{D wedge filling in pure systems}

A suitable starting point for the evaluation of the critical exponents is an effective interfacial Hamiltonian model based on a collective co-ordinate $l(\mathbf{x})$ measuring the height of the unbinding interface above the $z=0$ plane. At least for shallow tilt angles $\alpha \sim \tan \alpha$, the appropriate effective Hamiltonian is a natural generalization of the standard capillary-wave model used to study planar wetting and we write [7]

$$
H[l]=\int d \mathbf{x}\left\{\frac{\Sigma}{2}(\nabla l)^{2}+W(l-\alpha|x|)\right\}
$$

where $\Sigma$ is the stiffness coefficient of the unbinding interface which, for isotropic fluids, we can identify with the surface tension $\sigma_{l v}$. The model is not truly microscopic and is only valid for lengthscales much larger than the bulk correlation length. The binding potential $W(l)$ describes the influence of intermolecular forces and decays at large distances as $W(l) \sim-a l^{-p}$. The Hamaker constant $a$ is positive in the temperature region of interest whilst the value of exponent $p$ depends on the specific range of the forces with $p=2,3$ for non-retarded and retarded van der Waals forces respectively. It is also possible to modify the model to include the effect of quenched disorder but for the moment we concentrate on pure systems with thermal fluctuations. Minimisation of the capillary-wave-like model (7) determines the following mean-field values of the critical exponents for $3 \mathrm{D}$ filling $\underline{8}$

$$
\beta_{w}=\frac{1}{p} \quad, \quad \nu_{\perp}=\frac{1}{4} \quad, \quad \nu_{y}=\frac{1}{2}+\frac{1}{p}
$$

Thus fluctuation effects are extremely anisotropic at wedge filling with $\xi_{y} \gg \xi_{x}$ and are dominated by pseudo-one-dimensional local translations in the height of the filled region along the wedge 8, 9. We refer to these as "breather-mode" excitations. From the values of the above critical exponents it is clear that mean-field theory is only valid for intermolecular potentials with $p<4$ for which $\xi_{\perp} \ll l_{w}$. The filling fluctuation (FFL) regime corresponds to potentials with $p>4$ and to study this Parry, Rascón and Wood introduced a pseudo-one-dimensional wedge Hamiltonian which accounts only for the breather-mode excitations [8, 9]:

$$
H_{w}\left[l_{0}\right]=\int d y\left\{\frac{\Sigma l_{0}}{\alpha}\left(\frac{d l_{0}}{d y}\right)^{2}+V\left(l_{0}\right)\right\}
$$

where $l_{0}(y) \equiv l(0, y)>0$ is the local height of the interface above the wedge bottom and $l_{w}=\left\langle l_{0}(y)\right\rangle$. The model is considered valid only for small wave-vectors $k_{y} \ll k_{y}^{\max } \sim \xi_{x}^{-1}$. However because the fluctuations at filling are strongly anisotropic the relevant scaling combination $\xi_{y} k_{y}^{\max }$ diverges in the scaling limit and the cut-off does not determine universal quantities. The most important feature of this effective model is the presence of a bending term resisting fluctuations along the wedge which is proportional to the local interfacial height. At bulk coexistence the wedge potential $V\left(l_{0}\right)$ has the form

$$
V\left(l_{0}\right)=\frac{\Sigma}{\alpha}\left(\theta^{2}-\alpha^{2}\right) l_{0}+C l_{0}^{1-p}
$$


and for $\theta>\alpha$ has a minimum located at the mean-field value of $l_{w}$. Notice that the first term in the wedge binding potential is the small $\alpha$ approximation to the thermodynamic term $f_{w}$ and is proportional to the linear, temperature-like scaling field $t$. The transfer-matrix analysis of the one-dimensional model will be considered later. Here we point out two related interpretations of the model which give the desired classification of the allowed critical singularities without need for explicit calculations. Both arguments will play a role in our later discussions. To begin we observe that the bending energy term is invariant under a renormalization group rescaling

$$
y \rightarrow y^{\prime}=\frac{y}{b} \quad, \quad l_{0} \rightarrow l_{0}^{\prime}=\frac{l_{0}}{b^{\zeta} w}
$$

with wedge wandering exponent $\zeta_{w}=1 / 3$. Under this transformation the linear scaling field $t$ and wedge Hamaker constant $C$ rescale to $t^{\prime}$ and $C^{\prime}$ with

$$
t^{\prime}=b^{\frac{4}{3}} t \quad, \quad C^{\prime}=b^{\frac{4-p}{3}} C
$$

Thus as anticipated the intermolecular forces are relevant and irrelevant for $p<4$ and $p>4$ respectively. The temperature-like scaling field is always relevant and from its rescaling and the value of $\zeta_{w}$ we can identify the values of the exponents in the FFL regime as

$$
\beta_{w}=\frac{1}{4} \quad, \quad \nu_{\perp}=\frac{1}{4} \quad, \quad \nu_{y}=\frac{3}{4}
$$

Equivalently we may say that in the FFL the diverging lengths scale according to

$$
l_{w} \sim \xi_{\perp} \sim \xi_{y}^{\frac{1}{3}}
$$

which shows the dramatic influence the wedge geometry has on the fluid interfacial properties near the filling phase boundary. Closely related to this simple rescaling analysis is the construction of an effective potential. Heuristically we may imagine that the equilibrium interfacial height $l_{w}$ follows from minimization of an effective wedge potential which takes into account the contribution arising from the bending free energy. Noting the scaling relation between $l_{w}, \xi_{\perp}$ and $\xi_{y}$ together with the first term in the wedge Hamiltonian it is natural to suppose that the effective potential has the form

$$
V_{\text {eff }}\left(l_{0}\right)=V\left(l_{0}\right)+D l_{0}^{-\tau_{w}}
$$

where $\tau_{w}=2 / \zeta_{w}-3$ and the constant $D \propto \Sigma$. Thus for the present case of thermal disorder $\tau_{w}=3$ and the critical behaviour falls into two regimes for $p<4$ and $p>4$ depending on whether intermolecular forces or the bending energy is the leading order correction to the thermodynamic term.

It is straightforward to generalise these ideas to a $d$-dimensional wedge which has translational invariance in $d_{y}=d-2$ dimensions along the wedge. As we shall see consideration of the "compactifaction" from a three- to two-dimensional wedge highlights some interesting properties of the wandering exponent for planar interfaces. Breather-mode excitations do not lead to large-scale interfacial roughness for $d>4$ and mean-field theory is valid. For $d<4$ we find two possible fluctuation regimes corresponding to mean-field-like and fluctuation-dominated behaviour. The FFL occurs for sufficiently short-ranged potentials with $p>2\left(1 / \zeta_{w}-1\right)$ with a generalised wedge wandering exponent

$$
\zeta_{w}=\frac{4-d}{3}
$$


valid for $2<d<4$. The critical exponent for the divergence of the interfacial height in the FFL regime follows as

$$
\beta_{w}=\frac{4-d}{2(d-1)}
$$

and recovers the $1 / 4$ power law for the three-dimensional wedge. Intriguingly the result (17) has the correct two-dimensional limit $\beta_{w}=1$ for filling with thermal fluctuations. We shall return to this later but first focus on the 2D limit in more detail.

\subsection{Fluctuation-induced wedge covariance in $2 D$}

Interfacial Hamiltonian studies [10, 11, 12, reveal that the fluctuation regimes for 2D filling are different to those for $2 \mathrm{D}$ critical wetting. Nevertheless there appears to be a profound connection between the fluctuation-dominated regimes for each transition. Recall that critical wetting refers to the continuous unbinding of the liquid-vapour interface (say) from a planar wall as the temperature is increased to the wetting temperature $T_{\text {wet }}$ at bulk coexistence. The vanishing of the contact angle $\theta$ as $T \rightarrow T_{w e t}$ is accompanied by the divergence of the mean interfacial thickness $l_{\pi}$, roughness $\xi_{\perp}$ and transverse correlation length $\xi_{\|}$. More detailed information concerning the local height fluctuations is contained in the one-point interfacial height probability distribution function $(\mathrm{PDF}) P_{\pi}(l ; \theta)$ where, in preparation for our discussion of wedge covariance, we have written the PDF in terms of the contact angle. Wetting transitions are characterised by highly anisotropic fluctuations arising from the random-walk or capillary-wave-like motion of the interface which are quantified by

the scaling relation $\xi_{\perp} \sim \xi_{\|}^{\zeta(d)}$ with $\zeta(d)$ the wandering exponent for a free interface (see later). At bulk coexistence the critical exponents for critical wetting describing the asymptotic divergence of these lengthscales are identified from

$$
l_{\pi} \sim\left(T_{\text {wet }}-T\right)^{-\beta_{s}}, \xi_{\perp} \sim\left(T_{\text {wet }}-T\right)^{-\nu_{\perp}}, \xi_{\|} \sim\left(T_{\text {wet }}-T\right)^{-\nu_{\|}}
$$

Generically, fluctuation effects at critical wetting fall into three regimes depending by the range of the intermolecular forces 15, 16, 17, 18, 19. These are mean-field (MF), weak-fluctuation (WFL) and strong-fluctuation (SFL) scaling regimes. The SFL regime represents the universality class of critical wetting with sufficiently shortranged forces $p>2(1 / \zeta(2)-1)$ and is characterised by large fluctuations $l_{\pi} \sim \xi_{\perp}$ and universal critical exponents. In two dimensions rather general random-walk-based arguments predict that the critical exponents in the SFL regime are explicitly related to the value of the wandering exponent. In particular for the interfacial height it is believed that 17, 18,

$$
\beta_{s}=\frac{\zeta(2)}{1-\zeta(2)}
$$

which is certainly obeyed for pure $(\zeta(2)=1 / 2)$ and random-bond systems $(\zeta(2)=$ $2 / 3)$.

For 2D filling on the other hand interfacial Hamiltonian studies show there are only two fluctuation classes corresponding to mean-field and fluctuation-dominated regimes for which $l_{w} \gg \xi_{\perp}$ and $l_{w} \sim \xi_{\perp}$ respectively [10, 11, 12. In general the critical exponents describing mean-field filling and mean-field critical wetting transitions are unrelated. Also the filling fluctuation (FFL) regime, representing the universality class of systems with short-ranged forces, is broader than the SFL regime for critical wetting 
and occurs for $p>(1 / \zeta(2)-1)$. Simple scaling arguments indicate that within the FFL regime the interfacial height diverges with a critical exponent

$$
\beta_{w}=\frac{\zeta(2)}{1-\zeta(2)}
$$

which is in agreement with model calculations in pure [10] and impure [11] systems. Wedge covariance [12] refers to the "empirical" observation emerging from effective interfacial Hamiltonian and Ising model studies that the equality of the critical exponents also extends to the full scaling properties of the respective one-point PDF's. In brief, wedge covariance states that for systems with short-ranged forces, at bulk coexistence and in the scaling limit, the interfacial height PDF at the wedge mid-point $P_{w}(l ; \theta, \alpha)$ is identical to the analogous PDF for strong-fluctuation regime critical wetting at a planar wall-fluid interface but with an effective shifted contact angle $\theta \rightarrow \theta-\alpha$ with $\alpha$ the wedge tilt angle. Thus if $P_{\pi}(l ; \theta)$ denotes the critical wetting interfacial height PDF written in terms of the contact angle, wedge covariance implies

$$
P_{w}(l ; \theta, \alpha)=P_{\pi}(l ; \theta-\alpha)
$$

which is valid in the critical regime $\theta-\alpha \rightarrow 0$. From this it follows that the equilibrium mid-point height near a fluctuation-dominated filling transition satisfies

$$
l_{w}(\theta, \alpha)=l_{\pi}(\theta-\alpha)
$$

where, in an obvious notation, $l_{\pi}(\theta)$ denotes the SFL regime critical wetting film thickness expressed in terms of the contact angle. Interestingly, the covariance (21) necessarily leads to the exponent identifications (1920) whilst a third relation between the FFL wedge free energy and SFL point tension $\tau(\theta)$ leads to the new result for wetting [12]

$$
l_{\pi}(\theta)=-\frac{\tau^{\prime}(\theta)}{2 \sigma_{l v}}
$$

This expression leads directly to the identification of the critical singularity associated with the point tension consistent with exact Ising model calculations [20] and a more general conjecture due to Indekeu and Robledo [21. We emphasise that the above covariance relations, originally noted from interfacial Hamiltonian studies of filling in shallow wedges (in pure and impure systems), appear to be rather robust. For pure systems they are also obeyed by a drumhead interfacial model of filling in more acute wedges [22] and, most importantly, are consistent with exact [23] and numerical studies 24] of filling in the square lattice Ising model with tilt angle $\alpha=\pi / 4$.

\section{Classical Wedge Covariance for filling in shallow wedges}

The standard fluctuation theory of wetting at planar wall-fluid interfaces is based on analysis of effective interfacial Hamiltonian models which describe the fluctuations of a collective co-ordinate $l(\mathbf{x})$ measuring the local height of the interface from the wall. This coarse-grained description is valid at lengthscales much bigger than the bulk correlation length and, in its simplest form, is written

$$
H_{\pi}[l]=\int d \mathbf{x}\left\{\frac{\Sigma}{2}(\nabla l)^{2}+W(l)\right\}
$$

where, as described earlier, $\Sigma$ is the stiffness coefficient of the unbinding interface and $W(l)$ is the binding potential which, in general, accounts for the direct influence of 
intermolecular forces. We shall focus on isotropic fluid interfaces for which $\Sigma$ can be identified with the surface tension $\sigma_{l v}$.

For systems with short-ranged forces the binding potential describes not so much the range of the intermolecular potentials but the decay of perturbations in the local microscopic order parameter (density, magnetization) and is usually taken to have the form, at bulk coexistence,

$$
W(l)=-a e^{-\kappa l}+b e^{-2 \kappa l}
$$

where $\kappa$ is the inverse bulk correlation length of the wetting phase. The temperature dependence of the coefficients $a, b$ is crucial at mean-field level. The leading order term vanishes at the mean-field wetting temperature so that $a \propto\left(T_{\text {wet }}^{M F}-T\right)$ whilst $b$ must be positive finite at $T_{\text {wet }}$ to ensure stability and is usually taken to be constant. The structure of the binding potential can be inferred by comparison with more microscopic Landau-like mean-field theories. More formally it can derived from a constrained fluctuation sum as in the approach of Fisher and Jin which also leads to the presence of a position-dependent stiffness-coefficient [25], 26].

At mean-field level the equilibrium thickness $l_{\pi}$ of the interface follows from minimization of $W(l)$ whilst the contact angle $\theta$ can be identified from $\Sigma \theta^{2} / 2=$ $-W\left(l_{\pi}\right)$. Thus for systems with short-ranged forces the mean-field results are

$$
\kappa l_{\pi}=\ln 2 b / a \quad, \quad a=\sqrt{2 \Sigma b} \theta
$$

corresponding to critical exponents $\beta_{s}=0(\ln )$ and $\alpha_{s}=0$. Written in terms of the contact angle the equilibrium film thickness of the critical wetting layer is therefore

$$
\kappa l_{\pi}(\theta)=-\ln \sqrt{\frac{\Sigma}{2 b}} \theta
$$

which will be our intial point of reference for the wedge calculation.

For shallow wedges corresponding to tilt angles $\alpha \sim \tan \alpha$ the natural generalization of the planar model is the effective Hamiltonian (7) which simply assumes the interfacial interaction with the wall is controlled by the local relative height variable $\tilde{l}=l-\alpha|x|$. Note that the mean-field analysis is essentially independent of dimension since it assumes translational invariance along the wedge. The Hamiltonian is minimised subject to the appropriate boundary condition $\tilde{l} \rightarrow l_{\pi}$ as $|x| \rightarrow \infty$ and yields the Euler-Lagrange equation

$$
\Sigma l^{\prime \prime}(x)=W^{\prime}(l-\alpha|x|)
$$

It is convenient to write this in terms of the local relative height $\tilde{l}=l-\alpha|x|$ which, making analogy with classical mechanics, exploits the local Galilean invariance of the Euler-Lagrange equation. On integration one obtains the "energy" equation

$$
\frac{\Sigma}{2}\left(\left|l^{\prime}(x)\right|-\alpha\right)^{2}=\Delta W(l-\alpha|x|)
$$

where $\Delta W(l)=W(l)-W\left(l_{\pi}\right)$. Thus at the wedge mid-point one finds the simple expression for the local height of the filling film

$$
\frac{\Sigma}{2} \alpha^{2}=\Delta W\left(l_{w}\right)
$$

As pointed out by Rejmer et al [7] this equation has a very elegant graphical interpretation which demonstrates that for quite arbitrary choices of binding potential the wedge undergoes a filling transition when $\theta(T)=\alpha$, in precise accord with 
thermodynamic arguments. Now consider the specific case of short-ranged forces with the binding potential (25). Substitution determines the mid-point height as

$$
\kappa l_{w}(\theta, \alpha)=-\ln \sqrt{\frac{\Sigma}{2 b}}(\theta-\alpha)
$$

This not only identifies the logarithmic divergence of the film thickness at the filling transition, $\beta_{f}=0(\ln )$, but also reveals that the mean-field theory shows a "classical" analogue of wedge covariance observed in the $2 \mathrm{D}$ calculations:

$$
l_{w}(\theta, \alpha)=l_{\pi}(\theta-\alpha)
$$

In other words, for systems with short-ranged forces, the influence of the wedge geometry as manifest in the mid-point height is to shift the effective value of the contact angle. A similar property extends to the whole equilibrium profile which satisfies

$$
l(x)-\alpha|x|=l_{\pi}\left(\theta-\alpha+\left|l^{\prime}(x)\right|\right)
$$

and smoothly interpolates from $l_{\pi}(\theta-\alpha)$ to $l_{\pi}(\theta)$ as $|x|$ increases. Profile covariance indicates that the height dependence of the meniscus contains information about the contact angle dependence of the planar wetting film $l_{\pi}(\theta)$ and vice versa.

Classical wedge covariance is also manifest in the Gaussian fluctuations about the mean-field solutions. This is most easily interpretated for the 2D wedge. Consider for example the mean-field expression for the connected height-height correlation function

$$
S_{w}(0, x)=\langle(l(0)-\langle l(0)\rangle)(\tilde{l}(x)-\langle\tilde{l}(x)\rangle)\rangle
$$

and note that $S_{w}(0,0)=\xi_{\perp}^{2}$ identifies the mid-point roughness. The correlation function can be easily obtained from solution to the appropriate Ornstein-Zernike-like equation yielding

$$
S_{w}(0, x)=\frac{\left|\tilde{l}^{\prime}(x)\right|}{2 W^{\prime}\left(l_{w}\right)}
$$

where, for convenience, we have set $k_{B} T=1$. For systems with short-ranged forces the explicit result for the mean-field roughness at the wedge mid-point satisfies

$$
\xi_{\perp}(\theta, \alpha)^{2}=\frac{1}{2 \kappa \Sigma(\theta-\alpha)}
$$

This can be compared with the result pertinent to critical wetting at the planar wallfluid interface which can be obtained by simply setting $\alpha=0$,

$$
\xi_{\perp}(\theta)^{2}=\frac{1}{2 \kappa \Sigma \theta}
$$

implying the classical wedge covariance relation

$$
\xi_{\perp}(\theta, \alpha)=\xi_{\perp}(\theta-\alpha)
$$

for Gaussian fluctuations about the mean-field solution.

For higher-dimensional wedges the actual mid-point roughness $\xi_{\perp}$ is no longer covariant because of the breather-mode excitations along the wedge. For such dimensions the quantity analogous to the roughness which shows covariance is simply the zeroth moment of the the height-height correlation function

$$
S_{w}(0, x)=\int d y_{12}\langle(l(0)-\langle l(0)\rangle)(\tilde{l}(\mathbf{x})-\langle\tilde{l}(\mathbf{x})\rangle)\rangle
$$


where $y_{12}$ is the relative separation of the two points along the wedge. This satisfies the same expression (35) quoted above [8] and is therefore covariant.

We emphasise that classical covariance is not a general feature of the mean-field theory of filling and critical wetting. Within a more general description based on a binding potential

$$
W(l)=-a l^{-p}+b l^{-q}
$$

the filling and critical wetting exponents are distinct:

$$
\beta_{w}=\frac{1}{p} \quad, \quad \beta_{s}=\frac{1}{q-p}
$$

This automatically rules out the possibility of (classical) wedge covariance for binding potentials of the form (40). Nevertheless the results presented above showing wedge covariance for short-ranged forces do generalise to the class of potentials

$$
W(l)=-a \omega(l)+b \omega(l)^{2}
$$

where $\omega(l)$ corresponds to an arbitrary choice of monotonically decaying function. Choosing $\omega=e^{-\kappa l}$ we obtain the usual short-ranged binding potential whilst setting $\omega=l^{-p}$ one obtains a binding potential describing a particular type of multicritical wetting transition with $q=2 p$. Repeating the analysis above we obtain for the planar critical wetting and mid-point wedge filling interfacial heights

$$
\omega\left(l_{\pi}\right)=\sqrt{\frac{\Sigma}{2 b}} \theta \quad, \quad \omega\left(l_{w}\right)=\sqrt{\frac{\Sigma}{2 b}}(\theta-\alpha),
$$

which immediately implies the wedge-covariant relation (32). A little more algebra shows that the roughnesses also obey covariance implying that the mean-field PDF's are identical provided we map $\theta \rightarrow \theta-\alpha$. We refer to the class of potentials (42) as classical wedge covariant binding potentials. Of course, only for the short-ranged case $\omega=e^{-\kappa l}$ do we anticipate that such potentials have any physical significance. Nevertheless they do point to an important feature of the present analysis. It is easy to demonstrate that the wedge covariant potentials (42) all describe planar critical wetting transitions with vanishing specific heat exponent $\alpha_{s}=0$. This mirrors precisely the situation for the fluctuation-induced non-classical covariance since for $\zeta \geq 1 / 2$ the value of the specific heat exponent in the SFL regime is $\alpha_{s}=0$. This covers both cases, $\zeta=1 / 2$ and $\zeta=2 / 3$ in $2 \mathrm{D}$, where non-classical covariance is known to occur and it is tempting to speculate that the vanishing of the specific heat exponent either at mean-field level or beyond plays a key role for classical and non-classical covariance respectively.

The prediction of classical wedge covariance shows that this hidden relation between filling and wetting for special types of forces is not necessarily a fluctuationinduced phenomenon. Having said that we feel care should be taken in saying that the classical covariance is the underlying origin of non-classical covariance. Whilst they are certainly related it is far from obvious why the covariant relation (32) obeyed for binding potentials describing mean-field critical wetting with $\alpha_{s}=0$ should remain unaltered in the presence of large-scale fluctuation effects which renormalise the value of the exponent $\beta_{s}$ whilst leaving $\alpha_{s}$ unchanged. In view of this we treat classical covariance as a prediction which should be tested in more microscopic models of wetting and filling. Since the binding potential (25) is believed to describe wetting in systems with short-ranged forces we turn attention to numerical studies of filling based on a Landau-like density functional model. This will also allow to test any limitations arising from the shallow wedge approximation implicit in the interfacial model. 


\section{Wedge Filling within Landau Theory}

For our Landau theory study we resort to a magnetic terminology rather than the fluids-based one considered earlier. At mean-field level the equilibrium order parameter $m(\mathbf{r})$ is translationally invariant along the wedge so we can restrict ourselves to magnetization profiles in a two-dimensional space $\mathbf{r}=(x, z)$ with $x$ the co-ordinate across the wedge. The free-energy functional for the infinite wedge that we wish to minimise is

$$
F[m]=\int_{V} d \mathbf{r}\left\{\frac{1}{2}(\nabla m)^{2}-\frac{t}{2} m^{2}+\frac{u}{4} m^{4}-h m\right\}
$$

where the volume of integration is restricted to $z \geq \tan \alpha|x|$ for every $x$. The parameter $t$ measures the deviation from the bulk critical temperature (which is always finite) whilst $u>0$ for stability. The bulk field $h=0^{-}$so that the bulk magnetization is negative. The temperature dependence of the equilibrium profiles can be eliminated by measuring the magnetization in units of the bulk spontaneous magnetization $m_{0}(t)=\sqrt{t / u}$ and need not be specified further. Rather than use a local surface field and enhancement parameter we use fixed boundary conditions which set the surface magnetization to a positive value $m(x, \tan \alpha|x|)=m_{1}$ for all $x$. This is equivalent to the infinite surface enhancement limit in the model of Nakanishi and Fisher 27] and ensures that the wetting transition pertinent to the planar wall-down spin interface is always second-order. In the planar limit $\alpha=0$ the model can be solved analytically and exhibits a critical wetting transition when the surface magnetization

$$
m_{1}^{w e t}=m_{0}
$$

which allows us to induce wetting (and filling in the wedge) by either increasing $m_{1}$ at fixed $t$ or vary $t$ at fixed $m_{1}$. We have chosen to vary $m_{1}$ at fixed temperature since this keeps the bulk magnetization and correlation length $\xi_{b}=1 / \kappa=(2 t)^{-1 / 2}$ fixed. The contact angle within this model can be calculated analytically

$$
\cos \theta=\frac{3 m_{1}}{2 m_{0}}\left(1-\frac{m_{1}^{2}}{3 m_{0}^{2}}\right)
$$

so that near the wetting transition $\theta \propto\left(m_{0}-m_{1}\right) / m_{0}$ where $\left(m_{0}-m_{1}\right) / m_{0}$ may be regarded as the temperature-like linear scaling field. Thus in the wedge geometry it is straightfoward to convert the numerically-determined value of the surface magnetization at filling phase boundary $m_{1}^{\text {fill }}$ into a contact angle $\theta$. We also remark that near the filling transition the scaling field $\theta-\alpha$ is equivalent to $\left(m_{1}^{\text {fill }}-m_{1}\right) / m_{0}$.

We have numerically minimised a discretized version of the continuum Landau free-energy functional in a number of finite-size geometries with boundary conditions chosen to mimic the bulk as closely as possible. We considered a double wedge geometry with opposing surface magnetizations on the top and bottom wedges and also a capped wedge geometry (see Figure 21) with both free and fixed boundary conditions at $z=L_{1}$. The results for the phase boundary and logarithmic growth of the filling layer are the same in all of these geometries. After testing our algorithm on a number of standard one- and two-dimensional problems including the Laplace and Poisson equations we first applied the minimization scheme to the Landau freeenergy functional with $\alpha=\pi / 4$. We found that, as the system was made larger and the discretization finer, our results stabilised and the phase boundary could be made arbitrarily close to the theoretical prediction. The smallest system with the roughest 


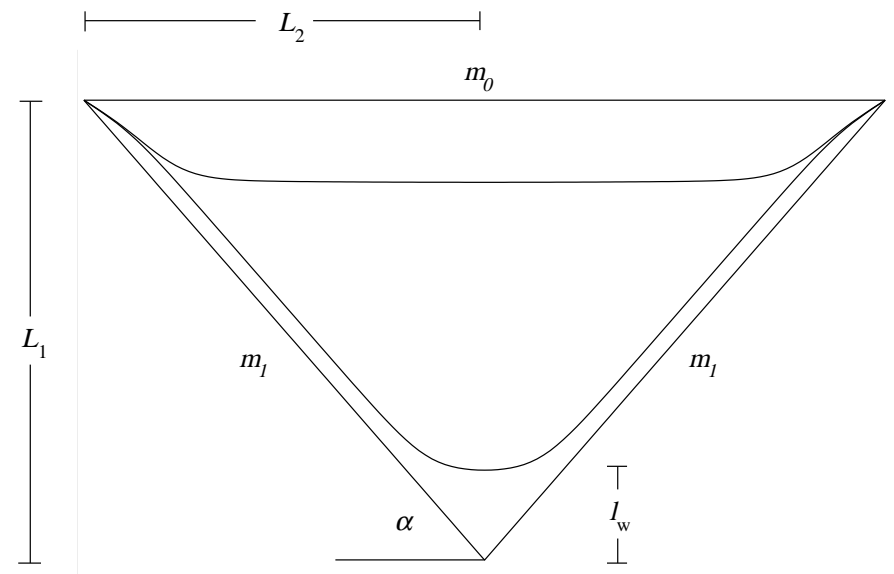

Figure 2. Typical capped wedge geometry used for the Landau numerical calculations. The magnetisation has a fixed value $m_{1}$ at the wedge boundaries and the bulk value $m_{0}$ at $z=L_{1}$. Here, $\alpha=45^{\circ}$ and $L_{1}=L_{2} \approx 30 \xi_{b}$. Two solutions corresponding to either side of a filling transition are shown: the lower interface for $m_{1} / m_{0}=0.5$ and the upper interface for $m_{1} / m_{0}=0.55$.

discretization that produced a clear logarithmic growth and a transition value of $\theta$ within 0.1 degrees of $\alpha$ was $L_{1}=L_{2} \approx 30 \xi_{b}$ and a distance between the grid points of approximately $\xi_{b} / 2$. Taking this as a starting point we increased the number of points on the grid and reduced the grid spacing in the vertical direction, producing a more open wedge of the same depth, with the same discretization in the horizontal direction and a finer discretization in the vertical. The number of points and the vertical scale were adjusted to produce the various wedge angles. A similar procedure was used to produce acute wedges, but here the horizontal distance $L_{2}$ was kept constant and the wedge made gradually deeper. This approach allowed us study filling in wedges with a fairly wide range of tilt angles, up to a maximum of about 70 degrees, in a reasonable amount of CPU time. The effect of the variable discretization can be seen in Figure 3 For the most open wedges, $\theta$ and $\alpha$ agree to four significant figures as the fine discretization in the direction of the film growth, whilst for the very acute wedges $\theta$ and $\alpha$ may differ by as much as 0.5 degrees.

The results of our study are presented in Figure 3 and Figure 4 In Figure 3 we present data for the numerically determined phase boundary for tilt angles between 15 and 75 degrees which is in excellent agreement with the theoretical prediction $\theta=\alpha$. We have also studied the meniscus profile defined as the surface of isomagnetization $m=0$ (see Figure 4). For wedges with tilt angles $\alpha \leq \pi / 4$ there is clear evidence for the logarithmic growth of the mid-point filling height

$$
\kappa l_{w}=A \ln \left(m_{1}^{\text {fill }}-m_{1}\right)+C
$$

with a universal, angle independent constant $A=1.02 \pm 0.04$ consistent with the shallow wedge limit $A=1$. The constant $C$ is non-universal as is the size of the asymptotic critical regime which decreases with increasing wedge angle. Nevertheless even for the most acute angles we consider there is no evidence that the amplitude $A$ 


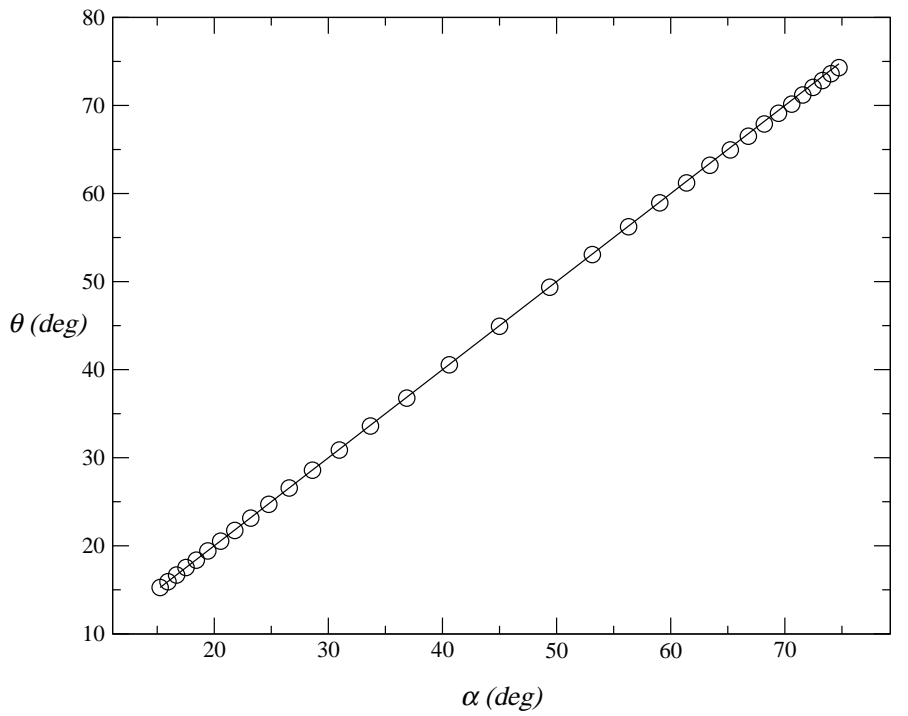

Figure 3. Plot of the contact angle at the filling transition, $\theta$, against the wedge angle, $\alpha$. The error bars on $\theta$ lie within the circles. The continuous line corresponds to the theoretical prediction Equation (5).

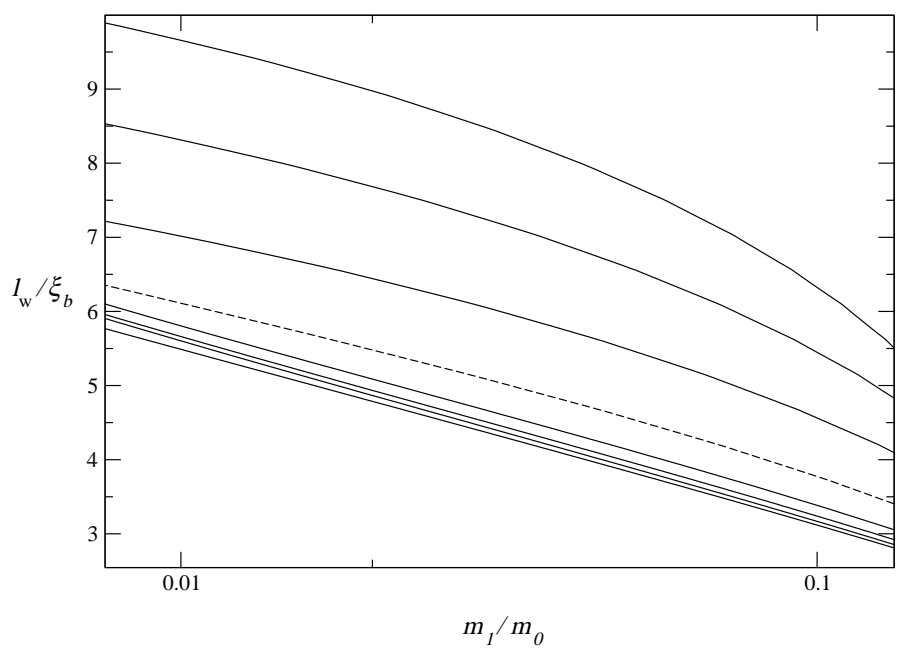

Figure 4. Plot of the reduced midpoint interface height, $l_{w} / \xi_{b}$, against the reduced surface magnetisation, $m_{1} / m_{0}$, for a range of $\alpha$ between approximately $15^{\circ}$ (bottom) and $75^{\circ}$ (top). The dashed line corresponds to $\alpha=45^{\circ}$. 


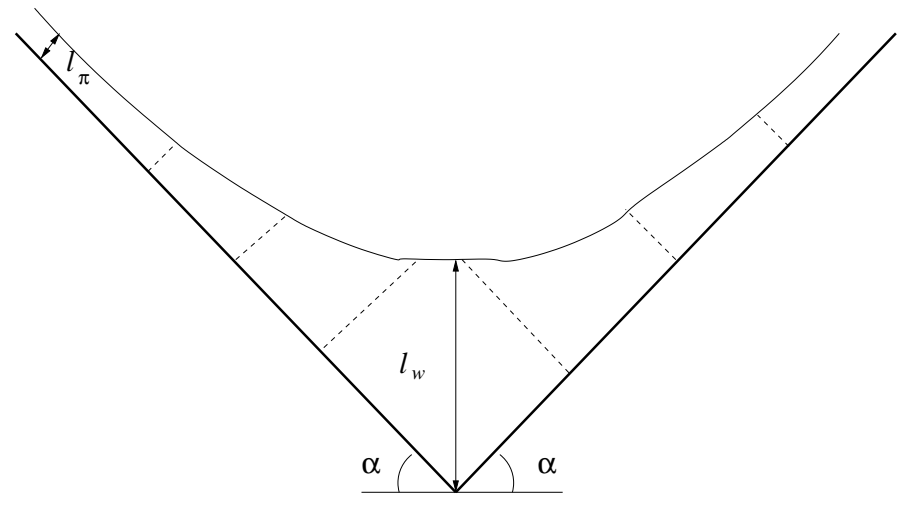

Figure 5. Substrate-interface interaction paths (dashed lines) for the wedge filling transition in the model of Rejmer et al $\mathbf{7}$.

differs from unity. More precisely for all wedge angles where there is clear logarithmic growth of the filling layer the amplitude of the logarithmic growth is consistent with $A=1$. In other words, even away from the shallow wedge limit the film diverges as

$$
\kappa l_{w}(\theta, \alpha) \sim-\ln (\theta-\alpha)
$$

and behaves, in the critical regime, precisely like $l_{\pi}(\theta-\alpha)$. This observation is certainly aesthetically appealing. In $2 \mathrm{D}$, all results point to non-classical covariance for both shallow and more acute wedges as indicated by exact Ising studies for the right-angle corner and also a drumhead model calculation with arbitrary wedge angle $\alpha$. The Landau theory numerics, coupled with the interfacial model studies for the shallow wedge, show that this is also the case for classical covariance.

Given that the interfacial model (77) is only valid for shallow wedges its is natural to inquire whether one can explain the Landau theory results using a generalised interfacial model valid for more acute wedges. We finish this section by showing that a very reasonable, and for many purposes satisfactory, drumhead-like interfacial model proposed by Rejmer, Dietrich and Napiórkowski (RDN) [7] does not fulfil this requirement. The model is a straightforward generalization of the shallow wedge Hamiltonian but is amended in two ways. First the square gradient term multiplying the tension is replaced by the correct expression for the total area of the liquid-vapour interface. This is familiar from older drumhead-like capillary-wave models and ensures that the model recovers the correct filling phase boundary $\theta=\alpha$. Secondly they propose replacing the relative vertical height from the wall to interface, appearing in the generalised binding potential contribution, by the normal distance to the closest wall (see Figure 5). Thus the RDN model for the 2D/3D wedge is given by [7]

$$
H_{R D N}=\int d \mathbf{x}\left\{\Sigma\left[\sqrt{1+(\nabla l)^{2}}-1\right]+\sec \alpha W(\sec \alpha[l-\psi(\mathbf{x}])\}\right.
$$

where, as earlier, $l=l(\mathbf{x})$ denotes the vertical height relative to the $\mathbf{x}=0$ plane and $\psi(\mathbf{x})=\tan \alpha|x|$. This assumption is certainly valid far from the wedge bottom where the interface and wall are parallel. One potentially unsatisfactory feature of it is that points on the wall for which for distances $|x|<l_{w} \sin \alpha \cos \alpha$ do not contribute 
to the binding potential i.e. the interface is blind to the wedge bottom. The meanfield equation for the mid-point height, obtained by minimising the Hamiltonian and integrating once, is

$$
\sigma_{l v}(\cos \theta-\cos \alpha)=W\left(l_{w} \cos \alpha\right)
$$

which is the analogue of the shallow wedge expression (28). In this way it is straightforward to see that the wedge filling transition occurs at the correct thermodynamic phase boundary $\theta=\alpha$ and that the critical exponents for continuous filling are independent of the wedge angle. Now consider the case of short-ranged forces modelled by the binding potential (25). According to the above drumhead model the asymptotic divergence of the mid-point height is given by

$$
\kappa l_{w} \sim-\sec \alpha \ln (\theta-\alpha)
$$

and contains a geometrical amplitude factor $\sec \alpha$ which is not consistent with the Landau theory numerics. In other words the RDN model does not give the correct, wedge-covariant result (48).

The failure of the RDN model to account for classical wedge covariance in more acute wedges is a surprising result. The model is certainly plausible and would appear to be the simplest possible generalization of the shallow wedge model. Indeed when considered beyond mean-field theory using transfer matrix methods, the RDN model does predict non-classical wedge covariance for $2 \mathrm{D}$ systems with strictly short-ranged forces! We emphasise that there is no mystery here; non-classical wedge covariance is a fluctuation-induced phenomenon for which the precise form of the binding potential (determining the way in which short-range forces are modelled) is irrelevant. In the presence of large fluctuations with $l_{w} \sim \xi_{\perp}$ all points on the interface can feel the influence of the wall through collisions with it. Thus in the 2D FFL regime it is sufficient to use a binding potential with a simple square well shape representing pure contact forces. This contrasts with classical wedge covariance which reflects the precise form of the underlying interfacial Hamiltonian model. At mean-field level there are no fluctuation effects that take the interface to the wall and even for short-ranged forces the manner in which one models the large distance exponential tail of the binding potential is crucial. Clearly the assumption that the wall-interface interaction occurs via the normal distance to points on the closest wall is incorrect. The absence of a $\sec \alpha$ prefactor in the Landau numerics (48) indicate that the correct measure of this is more akin to an effective local, vertical interaction similar to the shallow wedge model. This observation may well have ramifications for the construction of interfacial models of $3 \mathrm{D}$ wetting and filling in systems with short-ranged forces.

\section{3D wedge filling and the interfacial wandering exponent}

\subsection{Interfacial wandering and criticality at complete wetting}

In the treatment of fluctuation effects at 3D wedge filling presented in the introduction there appears to be little connection between the values of the critical exponents and the interfacial wandering exponent $\zeta(d)$ defined for a planar-like fluid interface. The value of the wandering exponent $\zeta(d)$ is crucial for discussions of depinning as well as complete and critical wetting transitions. Interfacial fluctuation effects at such transitions are isotropic in the $d-1$ dimensions parallel to the interface and 
the wandering exponent describes the scaling relation between the roughness $\xi_{\perp}$ and transverse or parallel correlation length $\xi_{\|}$[17:

$$
\xi_{\perp} \sim \xi_{\|}^{\zeta(d)}
$$

In general the value of $\zeta(d)$ depends on the dimensionality of space and the qualitative type of disorder. For pure systems, thermal fluctuations lead to interfacial roughness for $d \leq 3$ and we have the well-known result [17.

$$
\zeta(d)=\frac{3-d}{2}
$$

where for the marginal case, the identification $\zeta(3)=0$ corresponds to $\xi_{\perp} \sim \sqrt{\ln \xi_{\|}}$. The lower critical dimension for phase separation is $d_{L}=1$ at which $\zeta(d)=1$. The wandering exponent is altered in the presence of quenched impurities and it is usual to distinguish between random-field and random-bond disorder. Random fields induce interfacial roughness for $d<5$ and general scaling arguments lead to the result $\zeta(d)=(5-d) / 3$ [17, 28, 29] implying a lower critical dimension at $d_{L}=2$. For random bonds on the other hand the explicit dimension dependence of $\zeta(d)$ is not known. Near the upper marginal dimension, $d=5$, approximate functional renormalization group calculations of an interfacial model lead to the linear relation [17, 30]

$$
\zeta(d) \approx 0.2083(5-d)
$$

This is believed to be accurate even far from the marginal dimension since for $d=2$ it is close to the known exact result $\zeta(2)=2 / 3$ [31]. In three dimensions numerical results suggest $\zeta(3) \approx 0.44[32$, slightly higher than the value predicted by the linear relation (54). The lower critical dimension for interfacial wandering induced by random-bond disorder is $d_{L}=5 / 3$ [33], below which they are unstable with respect to thermal fluctuations.

The wandering exponent explicitly determines the fluctuation-dominated values of the critical exponents at the complete wetting transition. Complete wetting is pertinent to planar wall-vapour interfaces (say) with vanishing contact angle and refers to the divergence of the mean wetting layer (of liquid) thickness $l$, roughness $\xi_{\perp}$ and transverse correlation length $\xi_{\|}$as the bulk two-phase coexistence is approached. Writing the bulk ordering field $h \propto\left(\mu_{\text {sat }}(T)-\mu\right)$ we define critical exponents according to the power laws

$$
l \sim h^{-\beta_{s}^{c o}} \quad, \quad \xi_{\perp} \sim h^{-\nu_{\perp}^{c o}} \quad, \quad \xi_{\|} \sim h^{-\nu_{\|}^{c o}}
$$

The general theory of complete wetting is rather fully developed and can be understood using effective interfacial Hamiltonian models 34,35 . The usual starting point for the discussion of fluctuation regimes is the continuum model (7) with a binding potential

$$
W(l)=h l+a l^{-p}
$$

where the Hamaker constant $a$ is positive. Transfer matrix and renormalization group studies show that the critical behaviour falls in two scaling regimes [34. The values of the critical exponents in these regimes can also be understood using a simple effective potential picture. As noted by Lipowsky and Fisher 35] the form of the bending energy in the interfacial model suggests that fluctuation effects can be accounted for by minimising an effective potential

$$
W_{e f f}(l)=h l+a l^{-p}+b l^{-\tau}
$$




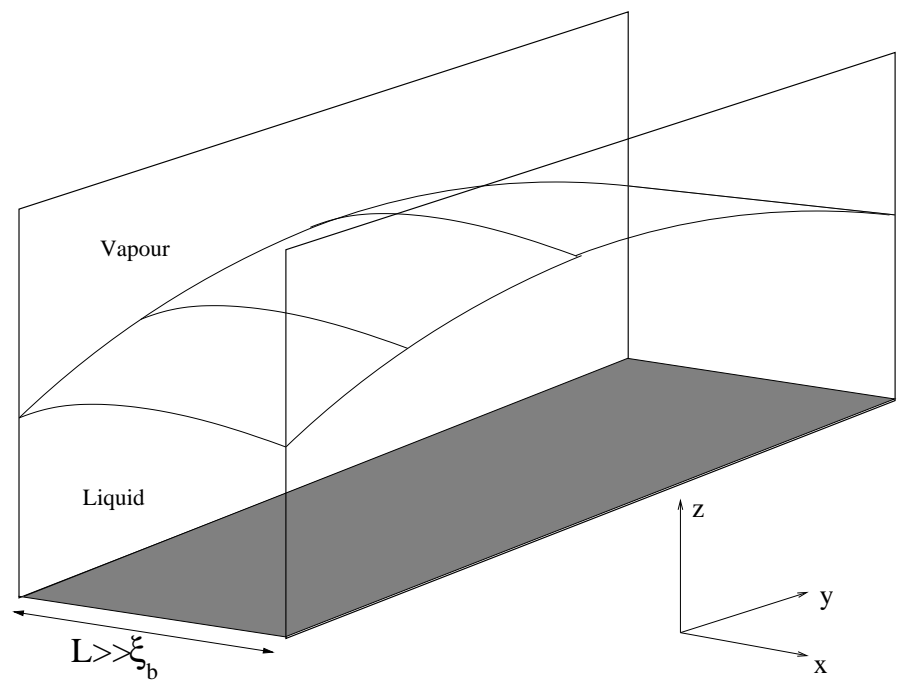

Figure 6. The vapour-liquid interface at complete wetting in a constrained geometry across the $x$-axis. See text for explanation.

where $\tau=2(1 / \zeta(d)-1)$. The complete wetting transition is therefore fluctuationdominated, belonging to the so-called weak-fluctuation (WFL) regime, for $\tau<p$ and leads to the critical exponent identification

$$
\beta_{s}^{c o}=\frac{\zeta(d)}{2-\zeta(d)}
$$

with $\beta_{s}^{c o}=\nu_{\perp}^{c o}=\zeta(d) \nu_{\|}^{c o}$. This approach together with the final exponent identification will be important in our discussion of filling in ordered and disordered systems.

\subsection{From complete wetting to filling}

Complete wetting and 3D filling share a number of common properties. Both transitions exhibit two fluctuation regimes reflecting the fact that there is always a relevant thermodynamic contribution to the appropriate effective potential. The difference in the values of the fluctuation repulsion exponents $\tau_{w}$ and $\tau$ can be traced directly to the extra lengthscale dependence in the effective stiffness at filling. A relation between these exponents can therefore be found if we understand how an additional lengthscale in the stiffness alters the fluctuation-induced repulsion. To this end consider a complete wetting transition occurring in a finite-size three-dimensional geometry with short-ranged forces (see Figure 6). The system is of infinite extent in the $y$ direction (say) but of finite width $L$ much bigger than the bulk correlation length. We may suppose that periodic boundary conditions apply across the system. In the presence of a finite bulk field $h$ the mean interface height is finite but diverges as $h \rightarrow 0$ with the growth of the film thickness falling into two regimes. If the width $L$ is larger than the transverse correlation length $\xi_{\|} \sim h^{-\nu_{\|}(3)}$ of a three-dimensional complete wetting layer of infinite extent, then the film grows with 3D-like critical exponents. If on the other hand $L \ll \xi_{\|}$the growth of the wetting layer thickness must be 
characterised by critical exponents pertinent to 2D complete wetting. Standard finitesize scaling ideas imply that the crossover between these regimes should emerge from the scaling hypothesis

$$
l(L, h)=h^{-\beta_{s}^{c o}(3)} \Lambda\left(L h^{\nu_{\|}(3)}\right)
$$

where $\Lambda(x)$ is an appropriate scaling function. In the limit $x \rightarrow \infty$ the scaling function must approach a constant consistent with the bulk 3D limit. On the other hand as the argument vanishes we must impose

$$
\Lambda(x) \sim x^{\frac{\beta_{s}^{c o}(3)-\beta_{s}^{c o}(2)}{\nu_{\|}^{(3)}}}
$$

in order to recover the correct 2D limit. Thus the asymptotic divergence of $l$ as $h \rightarrow 0$ contains the finite-size dependence

$$
l \sim h^{-\beta_{s}^{c o}(2)} L^{\frac{\beta_{s}^{c o}(3)-\beta_{s}^{c o}(2)}{\nu_{\|}^{(3)}}}
$$

This asymptotic divergence should also be understandable using a suitably modified effective potential measuring the free energy per unit length of the finite-size system. As $h \rightarrow 0$ the interface behaves as a 2D-like complete wetting layer in an effective bulk field $h_{e f f} \propto h L$ and with modified stiffness $\Sigma_{\text {eff }} \propto \Sigma L$. The divergence of $l$ should therefore follow from the minimization of an effective 2D-like complete wetting potential

$$
W_{e f f}(h, L)=(h L) l+A L^{-\phi} l^{-\tau(2)}
$$

where the exponent $\phi$ allows for the influence of the extra lengthscale dependence in the effective stiffness. In order to recover the correct finite-size result (61) we must identify

$$
\phi=\frac{\beta_{s}^{c o}(2)-\beta_{s}^{c o}(3)}{\beta_{s}^{c o}(2) \nu_{\|}(3)}+\frac{1}{\beta_{s}^{c o}(2)}-2
$$

This exponent is key to understanding the connection between interfacial wandering and 3D filling since it accounts for the correction to the standard fluctuation induced repulsion at wetting when the effective stiffness in the $y$-direction contains an additional length $\Sigma_{e f f}=\Sigma L$. This is also the case at filling where the effective stiffness along the wedge is proportional to the interfacial height $l_{w}$. Simple power counting implies that the fluctuation-induced repulsion exponent for the wedge is

$$
\tau_{w}=\phi+\tau(2)
$$

which reduces to

$$
\tau_{w}=\frac{2(1-\zeta(3))}{\zeta(2)}-1
$$

In this way we can explicitly relate all the $3 \mathrm{D}$ wedge filling exponents in the FFL regime to the values of the interfacial wandering exponent for $2 \mathrm{D}$ and $3 \mathrm{D}$ planar interfaces. Thus $l_{w} \sim \xi_{\perp} \sim \xi_{y}^{\zeta_{w}}$ with wedge wandering exponent

$$
\zeta_{w}=\frac{\zeta(2)}{(1+\zeta(2)-\zeta(3))}
$$

which is valid provided both the two- and three-dimensional interfaces are well-defined and rough. That is the dimensionality must be such that $d \leq d_{u}$ and $d-1>d_{L}$. 
Similarly, and subject to the same provisos, the critical exponent for the interfacial height reduces to

$$
\beta_{w}=\frac{\zeta(2)}{2(1-\zeta(3))}
$$

which is the central result of this section. The interpretation and implications of this result are discussed in detail below.

\subsection{Interpretation}

For pure systems the wandering exponent has values $\zeta(2)=1 / 2$ and $\zeta(3)=0$ in two and three dimensions respectively. Consequently (66) and (67) recover the effective Hamiltonian predictions $\zeta_{w}=1 / 3$ and $\beta_{w}=1 / 4$ discussed earlier. Further support for the connection between filling critical exponents and interfacial wandering arises when we generalise the above discussion to the $d$-dimensional wedge with $d-2$ dimensions along the direction of translational invariance. The analysis is unchanged except that $\zeta(2)$ and $\zeta(3)$ are replaced by $\zeta(d-1)$ and $\zeta(d)$ respectively. Thus for a generalised wedge we predict

$$
\beta_{w}=\frac{\zeta(d-1)}{2(1-\zeta(d))}
$$

provided that fluctuations are dominated by the $d-2$ dimensional breather modes along the wedge. Substituting the thermal result $\zeta(d)=(3-d) / 2$ recovers (17) for $d<3$. For $d>3$ (68) is no longer valid since the $d$-dimensional interface is no longer rough. Notice that, in the limit $d \rightarrow 2$, (68) recovers the correct two-dimensional filling result $\beta_{w}=1$ even though the breather-mode excitations are effectively zerodimensional, and that $\zeta(1)=1$.

For impure systems we are able to make some new predictions for filling in random-bond and random-field systems. For random-bond systems we use the values $\zeta(2)=2 / 3$ and $\zeta(3)=0.44$ to predict that in the FFL regime

$$
\beta_{w} \approx 0.60, \quad \nu_{\perp} \approx 0.60 \quad, \quad \nu_{y} \approx 1.1
$$

which are valid for sufficiently short-ranged forces. Assuming that there are only two fluctuation regimes and that there is a smooth cross-over from mean-field to fluctuation-dominated exponents we anticipate that the above predictions are valid provided the power law for the intermolecular forces $p>1.7$. Thus dispersion-like (van der Waals) forces are irrelevant in the renormalization group sense and belong to the universality class of systems with short-ranged forces. Note that the value of the fluctuation repulsion exponent $\tau_{w}$ is positive for both pure and random-bond systems.

The case of 3D filling in systems with random-field disorder is less clear-cut. Using the values $\zeta(2)=1$ and $\zeta(3)=2 / 3$ we are led to the predictions $\beta_{w}=\nu_{\perp}=3 / 2$ and $\nu_{y}=2$. However some caution is required in the assessing these predictions since the reliance on the marginal value $\zeta(2)=1$ is somewhat unsatisfactory. This situation is reminiscent of the two-dimensional limit of (68) for filling with thermal disorder which the relies on the marginal value $\zeta(1)=1$. The fact that the breather-mode picture generates the correct two-dimensional result for this case leads some support to the above predictions for 3D random-field filling. However the situation is worse for random-field disorder since the fluctuation repulsion exponent $\tau_{w}$ is negative for $d<10 / 3$. It is likely therefore that the exponent identification (67) is not appropriate 
for 3D wedge filling with random fields. Here after we limit our discussion to thermal and random-bond disorder only.

The above results for the values of the critical exponents for fluctuationdominated three-dimensional wedge filling in pure and impure (random-bond) systems complement the known result for two-dimensional filling (20). It is therefore interesting to ask how the three-dimensional result [67] becomes the two-dimensional result as we compactify the wedge and reduce the number of dimensions along it. For pure systems with only thermal disorder the situation seems straightforward since the result (68) is valid for $2 \leq d \leq 3$. As noted above the generalised breather-mode result smoothly recovers the correct numerical result $\beta_{w}=1$ in this limit. There is however something remarkable about this since equating the two expressions (20) and (68) implies $\zeta(2)=\zeta(1) / 2$ yielding information about the allowed value of the wandering exponent. Noting that the lower critical dimension for pure systems is $d_{L}=1$ and that at this dimension $\zeta=1$ we may conclude that equality of the breather-mode and wedge-covariant results for the critical exponents necessitates that for thermal fluctuations

$$
\zeta\left(d_{L}+1\right)=\frac{1}{2}
$$

which is correct. Turning attention to random-bond systems however note that we are not permitted to extend the breather mode result down to $d=2$ since the expression (68) is not valid when $d-1<d_{L}=5 / 3$ equivalent to $d<8 / 3$. Therefore we are not able to equate distinct expressions for the filling critical exponents in two dimensions and are unable to discuss the values of these exponents for $2<d<8 / 3$. However we are still free to discuss the properties of the filling critical exponents as $d \rightarrow 8 / 3$ from above. In particular, in view of the thermal result (70), it is natural to inquire what the value of the wandering exponent is for random-bond systems one dimension above the lower critical dimension. That is, what is the value of $\zeta(8 / 3)$ ? As discussed earlier a reliable estimate for the value of the wandering exponent is provided by the linear relation (54) which is only slightly below the exact result in two dimensions. At $d=8 / 3$ this yields $\zeta(8 / 3) \approx 0.49$ which we anticipate to be only slightly below the true result. This remarkable numerical coincidence leads us to conjecture that (70) is also valid for random-bond systems so that

$$
\zeta\left(\frac{8}{3}\right)=\frac{1}{2}
$$

It is natural to ask whether this conjecture has any support from other examples of fluctuating surfaces. Lipowsky has considered interfacial wandering and unbinding within a generalised class of effective Hamiltonians with bending energy term of the forms [15]

$$
H[l]=\frac{K}{2} \int d \mathbf{k} k^{2-\eta}|\tilde{l}(k)|^{2}+\int d \mathbf{x} W(l(\mathbf{x}))
$$

written in terms of the Fourier components of the collective co-ordinate. A remarkable property of such models is that for $\eta<0$ the fixed point behaviour (pertinent to short-ranged critical unbinding [36]) is controlled by a single parameter rather than by $\eta$ and $d$ separately. For our purposes we note only that the wandering exponent $\zeta(d)=(3-d-\eta) / 2$ and that the value at which this is unity allows us to identify $d_{L}=1-\eta$. This immediately implies that $\zeta\left(d_{L}+1\right)=1 / 2$ consistent with the known result for interfaces in pure systems and our conjecture for interfaces subject 


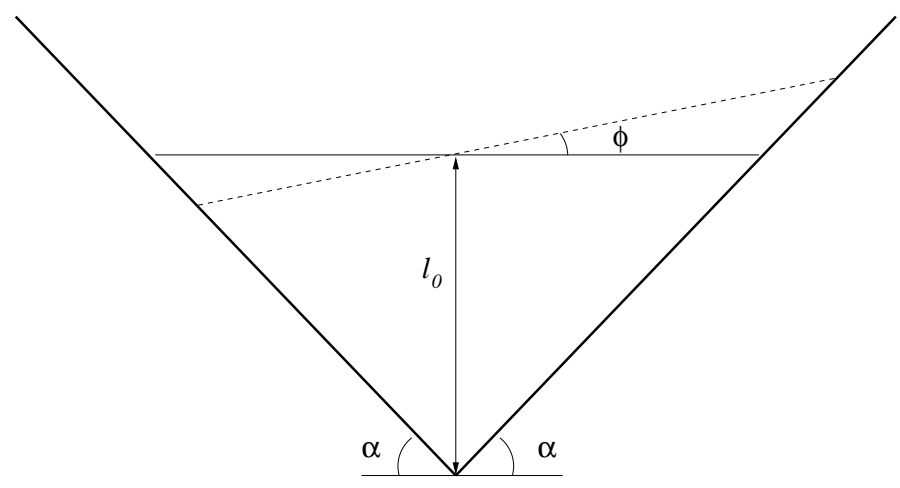

Figure 7. Schematic picture of the tilt fluctuations of the filled region in a section of a $3 \mathrm{D}$ wedge.

to random-bond disorder. On the other hand the result (70) is not universally valid since for interfaces in random fields $\zeta=(5-d) / 3$ implying $\zeta\left(d_{L}+1\right)=2 / 3$. Further work is required to substantiate the conjecture (71) for random-bond disorder.

\section{Tilt and torsional mode fluctuations at wedge filling}

In this final section we return to the problem of wedge filling in pure systems and reconsider the transfer matrix analysis of the wedge Hamiltonian (9). Bednorz and Napiórkowski [37] have pointed out that some ambiguities in the construction of the infinitesimal transfer matrix arise because the bending energy term is proportional to the interfacial height. To avoid this they propose that the fluctuating field or order parameter appearing in the partition function functional integration is not $l_{0}(y)$ but rather $z(y)=l_{0}(y)^{3 / 2}$. The advantage of choosing this field is clear since the bending energy term is a simple Gaussian and one can map the problem immediately onto Euclidean quantum mechanics. Here we point out that this choice of order parameter emerges naturally when we consider the role of tilt and torsional fluctuations coupled to breather-mode excitations.

Using the wedge Hamiltonian we wish to evaluate the partition function

$$
Z\left(l_{1}, l_{2} ; L\right)=\int D l_{0} e^{-\beta H_{w}\left[l_{0}\right]}
$$

for a wedge of length $L$ with fixed interfacial heights $l_{1}=l(0)$ and $l_{2}=l(L)$ at the end points. The measure $D l_{0}$ denotes some suitable integration over the allowed values of the collective co-ordinate $l(y)$ and will be discussed further below. The wedge model (9) was constructed on the assumption that the only fluctuations that are relevant for filling correspond to local translations in the height of the filled region. Let us generalise this and allow for small, local tilts in the flat, filled region of the interface, that mimic the effect of the intrinsic capillary fluctuations around the constrained profile. That is we consider a restricted class of interfacial configurations, that within the filled region has the form

$$
l(x, y)=l_{0}(y)+\phi(y) x \quad ; \quad l(x, y)<\alpha|x|
$$


provided $l(x, y)<\alpha|x|$. This is shown schematically in Figure 7 The tilt angle is assumed to lie in some interval $-\epsilon \alpha \leq \phi \leq \epsilon \alpha$ with $0<\epsilon<1$. Outside of the filled region we assume that the interface remains within a microscopic distance from the wall so that $l(x, y) \approx \alpha|x|$. Substitution into the capillary-wave model leads to a wedge Hamiltonian that allows for breather, tilt and torsional modes and is a functional of the two fields $l_{0}$ and $\phi$. It is straightforward to show that the breather-tilt-torsional (BTT) model has the form

$$
\begin{aligned}
& H_{B T T}\left[l_{0}, \phi\right]=\int d y\left\{\frac{K_{1}}{2} l_{0}\left(\frac{d l_{0}}{d y}\right)^{2}+K_{2} l_{0}^{2} \phi\left(\frac{d l_{0}}{d y}\right)\left(\frac{d \phi}{d y}\right)\right. \\
& \left.+\frac{K_{3}}{2} l_{0}^{3}\left(\frac{d \phi}{d y}\right)^{2}+V\left(l_{0}, \phi\right)\right\}
\end{aligned}
$$

where the generalised wedge potential is

$$
V\left(l_{0}, \phi\right)=\frac{\Sigma}{\alpha}\left(\theta^{2}-\alpha^{2}+\phi^{2}\right) l_{0}+C l_{0}^{1-p}
$$

The bending energy coefficient for the breather mode $K_{1}=\frac{2 \Sigma}{\alpha}$ and is unchanged from the simpler model (9). The coefficients appearing in the torsional terms are $K_{2} \propto \Sigma \alpha^{-2}$ and $K_{3} \propto \Sigma \alpha^{-3}$ respectively. The precise values of these coefficients are not important in the critical regime. It is also convenient to re-write the wedge potential as

$$
V\left(l_{0}, \phi\right)=t l_{0}+C l_{0}^{1-p}+\frac{\Sigma}{\alpha} \phi^{2} l_{0}
$$

Notice that when we set the field $\phi(y)=0$ at all points along the wedge the BTT Hamiltonian reduces to the wedge model (85). To assess the relevance of tilt and torsional modes on the critical behaviour of the model we consider a rescaling of the co-ordinates and fields similar to that discussed earlier. With the additional tilt field we now consider the properties of the model under the mapping

$$
y \rightarrow y^{\prime}=\frac{y}{b} \quad, \quad l_{0} \rightarrow l_{0}^{\prime}=\frac{l_{0}}{b^{\zeta_{w}}} \quad, \quad \phi \rightarrow \phi^{\prime}=\phi b^{2 \zeta_{w}}
$$

where, as before, the wedge wandering exponent $\zeta=1 / 3$. Under this rescaling the coefficient of the breather-mode bending coefficient $K_{1}$ and the term $\phi^{2} l_{0}$ appearing in the wedge potential remain invariant. The linear temperature-like scaling field $t$ and Hamaker constant $C$ rescale as earlier (see (12) ) whilst the new tilt and torsional bending energy coefficients renormalise to

$$
K_{2}^{\prime}=b^{-4 / 3} K_{2} \quad, \quad K_{3}^{\prime}=b^{-4 / 3} K_{3}
$$

and are therefore irrelevant for all ranges of intermolecular force. This means that they do not affect the critical behaviour and that the BTT model can be simplified to

$$
H_{B T T}\left[l_{0}, \phi\right]=\int d y\left\{\frac{\Sigma l_{0}}{\alpha}\left(\frac{d l_{0}}{d y}\right)^{2}+V\left(l_{0}, \phi\right)\right\}
$$

The tilt field $\phi$ is therefore non-interacting but still coupled to the breather-mode excitations. As the interface unbinds from the wedge bottom the tilt fluctuations become increasingly massive and are suppressed. An important consequence of this is that when we integrate out the tilt field the particular choice of the parameter $\epsilon$ becomes unimportant in the scaling limit. 
The final ingredient in the construction of the model is the nature of the measure $D l_{0} D \phi$ appearing in the functional integration over the Boltzmann weight $e^{-\beta H_{B T T}[l, \phi]}$, where $\beta \equiv 1 / k_{B} T$. In reducing the dimensionality of the collective coordinate $l(x, y) \rightarrow(l(y), \phi(y))$ some care is required in defining the new measure since we must impose the correct normalization conditions. This is most easily illustrated for 2D wedge filling in which there is no $y$-direction and the choice of measure $D l_{0} D \phi$ relates only to one $l$ and one $\phi$ variable. For this case the Boltzmann weight for a tilt configuration is simply $e^{-\beta V(l, \phi)}$ and determines the un-normalised probability $P\left(l_{0}, \phi\right)$ for finding an interface with tilt angle $\phi$ and mid-point height $l_{0}$. Integrating over the allowed values of $\phi$ determines the mid-point height PDF $P\left(l_{0}\right)$. The properties of this PDF are known from exact calculations. In particular for shortranged forces (belonging to the filling fluctuation regime) the function has the simple exponential form $P\left(l_{0}\right) \propto e^{-2 \beta \Sigma(\theta-\alpha) l_{0}}$ in the scaling limit. Thus we require that as $\theta \rightarrow \alpha$

$$
e^{-2 \beta \Sigma(\theta-\alpha) l_{0}} \sim \int_{-\epsilon \alpha}^{\epsilon \alpha} D l_{0} d \phi e^{-\beta \Sigma\left(\theta^{2}-\alpha^{2}+\phi^{2}\right) l_{0} / \alpha}
$$

which necessitates that we interpret the $2 \mathrm{D}$ measure $D l_{0} \propto \sqrt{l_{0}} d l_{0}$. In other words the correctly normalised PDF $P\left(l_{0}, \phi\right) \propto \sqrt{l_{0}} e^{-\beta V(l, \phi)}$. An equivalent argument is that after integrating over the tilt fluctuations the resulting PDF $P\left(l_{0}\right)$ must have the correct short-distance expansion as the interface approaches the wall. For $2 \mathrm{D}$ systems with short-ranged forces the PDF has the short-distance expansion $P\left(l_{0}\right) \sim l_{0}^{\gamma}$ with $\gamma=1 / \beta_{w}-1$. Recall that for pure systems $\beta_{w}=1$ (in 2D) implying that the twofield PDF $P\left(l_{0}, \phi\right)$ must contain a $\sqrt{l_{0}}$ prefactor in-order to preserve thermodynamic consistency. This argument is supported by explicit results for the $2 \mathrm{D}$ wedge filling for contact binding potentials (see Appendix A).

A similar line of reasoning applies in higher dimensions. To determine the form of the 3D measure we first focus on the filling transition occurring in a cone geometry. The reason for this is that the breather-mode excitations are essentially zero-dimensional and we only have one height variable $l_{c}$ measuring the height of the interface above the cone vertex. Up to a normalization factor the probability of finding the interface at height $l_{0}$ with tilt $\phi$ is determined by the Boltzmann weight $e^{-\beta V_{c}}$ where $V_{c}$ is the free-energy cost of the configuration. The leading order term in the cone binding potential contains a term proportional to the area $l_{0}^{2}$ of the filled region. The prefactor of this is $\beta \Sigma\left(\theta^{2}-\alpha^{2}+\phi^{2}\right)$ similar to the $2 \mathrm{D}$ wedge and integrating over the tilt modes (and a trivial rotational degree of freedom) determines the mid-point height PDF $P_{c}\left(l_{c}\right)$. In order to preserve the simple Gaussian form of this function we must interpret the 3D measure $D l_{c} \propto l_{c} d l_{c}$. For the 3D wedge we discretise the system in the $y$-direction and replace the fields $\{l(y)\}$ and $\{\phi(y)\}$ by sets of continuous variables $l_{j}$ and $\phi_{j}$. The wedge measure must be proportional to a product of cone measures and thus we interpret the functional integral for the BTT Hamiltonian as

$$
\int D l_{0} D \phi \propto \iiint \prod_{j} l_{0}(j) d l_{0}(j) d \phi_{j}
$$

On integrating over each tilt angle we recover the wedge model (9) and identify the functional integral appearing in (73) as the continuum limit of

$$
\iint \cdots \int \prod_{j} l_{0}(j)^{1 / 2} d l_{0}(j)
$$


In terms of the variable $z_{j}=l_{0}(j)^{3 / 2}$ the functional integral therefore becomes

$$
\iint \ldots \int \prod_{j} d z(j)
$$

and has a well-defined infinitesmal transfer matrix limit. In terms of the new variable the continuum wedge Hamiltonian is

$$
\tilde{H}_{w}[z]=\int d y\left\{\frac{4 \Sigma}{9 \alpha}\left(\frac{d z}{d y}\right)^{2}+\tilde{V}(z)\right\}
$$

where $\tilde{V}(z) \equiv V\left(z^{2 / 3}, 0\right)$. The rest of the analysis is identical to that described by Bednorz and Napiórkowski [37] and the problem can be mapped immediately on to quantum mechanics. Thus the partition function may be written as the spectral expansion

$$
Z\left(l_{1}, l_{2} ; L\right)=\sum_{n} \psi_{n}\left(l_{1}\right)^{*} \psi_{n}\left(l_{2}\right) e^{-\beta E_{n} L}
$$

where the eigenfunctions and eigenvalues satisfy the Schrödinger equation

$$
-\frac{9 \alpha}{16 \beta^{2} \Sigma} \psi_{n}(z)^{\prime \prime}+\tilde{V}(z) \psi_{n}(z)=E_{n} \psi_{n}(z)
$$

with boundary conditions $\psi(0)=\psi(\infty)=0$. Hence the PDF for finding the interface at height $l_{0}$ can be identified with $P_{w}\left(l_{0}\right)=\left|\psi_{0}\left(l^{3 / 2}\right)\right|^{2}$ whilst the wedge free energy and correlation length follow from $f_{w}=E_{0}$ and $\xi_{y}^{-1}=\beta\left(E_{1}-E_{0}\right)$ respectively. For the choice of wedge potential (10) it is straightforward to deduce the existence of two fluctuation regimes with the critical exponents discussed earlier.

Our final remarks concern the form of the interfacial height PDF $P\left(l_{0}\right)$ in the FFL regime. The intermolecular forces are irrelevant for $p>4$ and in the limit $\theta \rightarrow \alpha$ the scaling properties of the PDF follow from solution of the differential equation

$$
-\frac{9 \alpha}{16 \beta^{2} \Sigma} \psi_{0}(z)^{\prime \prime}+2 \Sigma(\theta-\alpha) z^{\frac{2}{3}} \psi_{0}(z)=E_{0} \psi_{0}(z)
$$

It follows immediately that the wave-function and hence PDF are scaling functions of the dimensionless variable $u=\sqrt{\beta \Sigma}(\theta / \alpha-1)^{1 / 4} l_{0}$ and we can write

$$
P\left(l_{0}\right)=\sqrt{\beta \Sigma}\left(\frac{\theta}{\alpha}-1\right)^{1 / 4} g\left(\sqrt{\beta \Sigma}(\theta / \alpha-1)^{1 / 4} l_{0}\right)
$$

where $g(u)$ is a suitably normalised scaling function such that $\int d u g(u)=1$. The scaling of the PDF is, of course, to be anticipated since in the FFL both $l_{w}$ and $\xi_{\perp}$ diverge with the same critical exponent. We are not aware of a closed form solution to the differential equation in terms of elementary functions. However both the asymptotic short-distance and large-distance behaviours agree with thermodynamic and sum-rule requirements. First consider the large distance limit corresponding to $u \gg 1$. From solution of (88) it is straightforward to show that the PDF decays like

$$
P\left(l_{0}\right) \sim \sqrt{\beta \Sigma}(\theta / \alpha-1)^{1 / 4} e^{-2 \beta \Sigma \sqrt{2(\theta / \alpha-1)} l_{0}^{2}}
$$

up to terms of order $l_{0}$ in the argument. This is precisely in keeping with macroscopic requirements. When the interface is far from the wall the PDF can be identified with $e^{-\beta \Delta F}$ where $\Delta F$ is the free-energy cost of a macroscopic configuration constrained to pass through the height $l_{0}$. In the wedge geometry this macroscopic configuration has the shape shown in Figure 7 and the free-energy cost is dominated by a thermodynamic 


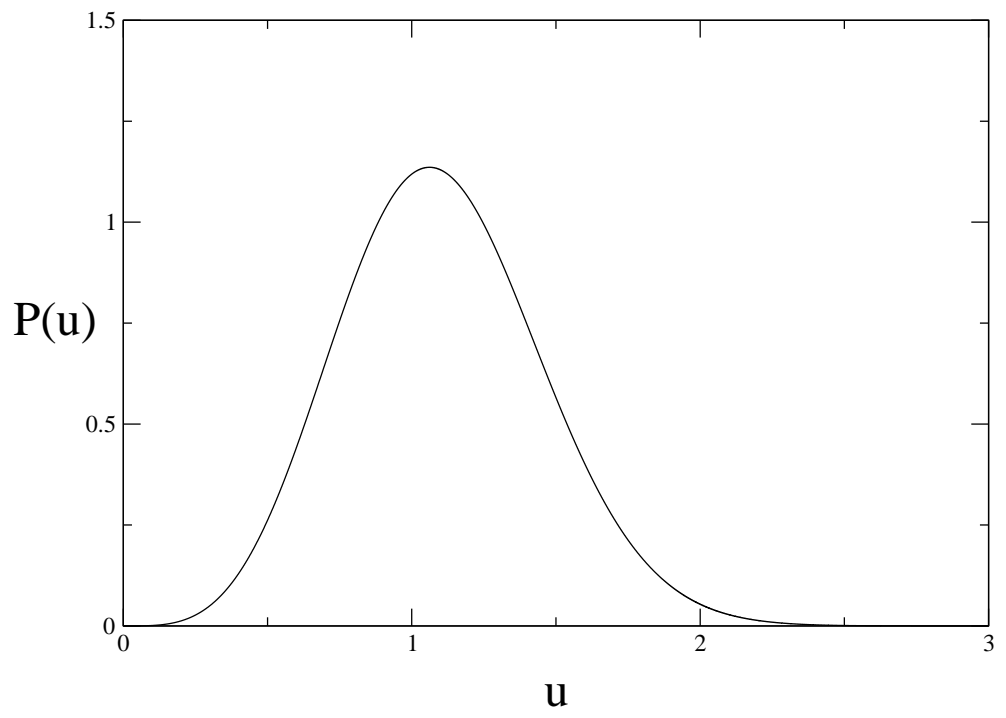

Figure 8. Rescaled probability distribution function for the interfacial height above the wedge bottom found from the breather mode model. Integration of the PDF determines the density profile in the wedge.

area-like contribution. This can be readily calculated making use of Young's equation yielding $\Delta F=2 \Sigma \sqrt{2(\theta-\alpha) / \alpha} l_{0}^{2}$. The short-distance expansion (SDE) of the scaling function $g(u)$ corresponding to the limit $u \ll 1$ is also consistent with known critical exponent relations. For 2D and 3D wedge filling the scaling function for the PDF has the short-distance behaviour

$$
g \sim u^{\gamma}
$$

where the SDE critical exponent $\gamma=1 / \beta_{w}-1$. This exponent relation follows from rather general sum-rule and scaling arguments. Thus for 3D filling in pure systems with $\beta_{w}=1 / 4$ we anticipate

$$
P\left(l_{0}\right) \sim(\beta \Sigma)^{2}\left(\frac{\theta}{\alpha}-1\right) l_{0}^{3}
$$

for $\sqrt{\beta \Sigma}(\theta / \alpha-1)^{1 / 4} l_{0} \ll 1$. This emerges naturally from solution to the Schrödinger equation since in terms of the $z$ variable $\psi(z) \sim z$ as $z \rightarrow 0$ and recall $P\left(l_{0}\right)=$ $\left|\psi\left(l_{0}^{3 / 2}\right)\right|^{2}$. The large-distance and SDE properties of the PDF give strong support to the correctness of the one-dimensional wedge Hamiltonian model. The numerically determined scaling form of the PDF found from solution to the Schrödinger equation is shown in Figure 8

\section{Conclusions}

In this paper we have addressed a number of issues pertaining to 3D wedge filling transitions. We began with a discussion of wedge covariance relations between filling and critical wetting and showed that the fluctuation-induced covariance observed for 2D filling has a mean-field analogue. Similar to the 2D case classical covariance 
appears to be specific to systems for which the critical wetting specific heat exponent $\alpha_{s}=0$. Numerical studies of mean-field filling within a Landau theory show that classical covariance is not limited to the shallow wedge limit. Importantly this provides a constraint on the possible form of effective Hamiltonian models of wedge filling and implies that a model suggested by Rejmer et al [7] is not consistent with more microscopic approaches. It is likely that in order capture the correct classical covariance for wetting and filling it will be necessary to construct non-local interfacial models. This will be discussed elsewhere.

In the second part of our paper we turned attention to fluctuation effects at $3 \mathrm{D}$ wedge filling. Our central new result is that the critical exponents which characterise the fluctuation-dominated regime can be related to the values of the standard wandering exponent for planar interfaces in 2D and 3D (bulk) systems. The expressions for $\beta_{w}$ and the wedge wandering exponent $\zeta_{w}$ expressed in terms of $\zeta(2)$ and $\zeta(3)$ are rather general and allow the discussion of filling in systems with random-bond disorder. A surprising feature emerging from our study concerns some intriguing numerical coincidences for the value of the bulk wandering exponent $\zeta(d)$ as one approaches the lower marginal dimension for wedge filling in pure and randombond systems. In particular we are led to conjecture that for random-bond disorder $\zeta(8 / 3)=1 / 2$.

In the final part of our paper we addressed some queries that have been highlighted concerning the pseudo-one-dimensional wedge Hamiltonian theory of 3D filling in pure systems. Bednorz and Napiórkowski [37 have suggested that these can be avoided if one supposes that the fluctuating field is not the interfacial height $l_{0}(y)$ above the wedge bottom but $l_{0}(y)^{3 / 2}$. We showed that this choice indeed emerges naturally if one considers the coupling between tilt and torsional fluctuations described by a second field $\phi(y)$ and breather-mode excitations of $l_{0}(y)$. The form of the PDF for the interfacial height predicted by the wedge Hamiltonian model is shown to have the correct short-distance and large-distance behaviour dictated by macroscopic arguments and critical exponent relations. We believe this strongly supports the internal consistency of the effective wedge Hamiltonian model. As mentioned in the introduction the values of the critical exponents predicted by the wedge Hamiltonian are in very good agreement with Ising model simulation studies. A more stringent test of the theory would be to study the scaling of the interfacial height PDF. This can be readily extracted from Ising model studies since it corresponds to the derivative of the magnetization profile measured along the vertical above the wedge bottom. To do this quantitatively it would be necessary to generalise the present theory to study the finite-size scaling of the PDF and magnetization profile in the same geometry studied by Milchev et al 13, 14. This will be a topic of future work.

Finally we mention that ideally one would hope to circumvent the entire wedge Hamiltonian theory and replace it with a more microscopic approach. In particular it would be extremely useful if one could apply a renormalization group analysis to derive the wedge filling critical exponents from the full capillary-wave model (7). To do this one would have to identify a new fixed-point distinct from the Gaussian (or strongfluctuation regime) fixed-point used to study wetting transitions. Such additional fixed points for the capillary-wave model may exist if one generalises the spatial rescaling to account for the anisotropy of correlation lengths across and along the wedge. One would hope, of course, that such an approach would lead to the same predictions for universal critical exponents and scaling functions found using the effective onedimensional wedge Hamiltonian. As well as being an independent check on the validity 
of the wedge Hamiltonian model (in the scaling limit) such an approach would also shed much light on the emergence of an effective one-dimensional theory from a higher dimensional model.

\section{Acknowledgments}

The authors wish to thank Dr A. J. Wood for discussions concerning the nature of wedge filling in disordered systems, and I-D Media AG, Berlin, for the donation of a workstation. MJG and JMR-E gratefully acknowledge the EPSRC and Secretaría de Estado de Educación y Universidades (Spain), co-financed by the European Social Fund for financial support respectively.

\section{Appendix A. Tilt mode fluctuations at 2D wedge filling}

In this Appendix we provide further justification for the role played by tilt fluctuations at $2 \mathrm{D}$ wedge filling making contact with exact results obtained from interfacial Hamiltonian studies. We restrict attention to systems with short-ranged forces corresponding to the FFL regime and set $k_{B} T=1$ for convenience. Consider the 3 -point probability distribution function $P_{w}\left(l_{1},-x ; l_{0}, 0 ; l_{2}, x\right)$ determining the probability for finding the interface at heights $l_{1}, l_{0}$ and $l_{2}$ at positions $-x, 0$ and $x$, respectively. Exact transfer-matrix results for a continuum interfacial model of filling in shallow wedges show that $P_{w}\left(l_{1},-x ; l_{0}, 0 ; l_{2}, x\right)$ decomposes into the product 38.

$$
P_{w}\left(l_{1},-x ; l_{0}, 0 ; l_{2}, x\right)=P_{w}\left(l_{0}\right) P_{\pi}^{c}\left(\tilde{l}_{1}, x \mid l_{0}, 0\right) P_{\pi}^{c}\left(\tilde{l}_{2}, x \mid l_{0}, 0\right)
$$

where $P_{w}\left(l_{0}\right)=2 \Sigma(\theta-\alpha) \exp \left[-2 \Sigma(\theta-\alpha) l_{0}\right]$ is the wedge midpoint 1 -point PDF, $\tilde{l}=l-\alpha x$ is the local relative height to the wall (recall that $x$ is positive) and $P_{\pi}^{c}\left(l_{2}, x_{2} \mid l_{1}, x_{0}\right)$ is the planar conditional probability distribution function [38]:

$$
\begin{aligned}
& P_{\pi}^{c}\left(l_{2}, x \mid l_{1}, 0\right)=\sqrt{\frac{\Sigma}{2 \pi x}} \mathrm{e}^{-\frac{\Sigma\left(l_{2}-l_{1}+\theta x\right)^{2}}{2 x}}+\mathrm{e}^{-2 \Sigma \theta l_{2}}\left[\sqrt{\frac{\Sigma}{2 \pi x}} \mathrm{e}^{-\frac{\Sigma\left(l_{1}+l_{2}-\theta x\right)^{2}}{2 x}}\right. \\
& \left.+\Sigma \theta \operatorname{erfc}\left(\sqrt{\frac{\Sigma}{2 \pi x}}\left(l_{1}+l_{2}-\theta x\right)\right)\right]
\end{aligned}
$$

If the wedge midpoint height is much greater than the mean wetting height for a planar substrate, i.e. $l_{0} \gg 1 / 2 \Sigma \theta$, which is fulfilled in the asymptotic critical regime and the position $x<l_{0} / \theta$, the second term in Equation (A.2) can be neglected and the conditional probability distribution function becomes a gaussian distribution of average $l_{0}-\theta x$ and dispersion $\sigma^{2}=x / \Sigma$. The interface will be affected by the presence of the substrate when $l_{0}-\theta x \sim \sqrt{x / \Sigma}$. This expression defines the effective value of $x^{*}$ at which the interface is in contact with the substrate. We consider the angle formed by the horizontal and the line that joins the interface position at the midpoint and the position (relative to the substrate) at $x^{*}$ (see Figure A1). The tilt angles $\Delta \theta_{1}$ and $\Delta \theta_{2}$ are defined as the departures of the previously defined angles with respect to the average value $\theta$. We stress that the interface is not considered stiff but is allowed to wander due to capillary-wave fluctuations. However, the length scale on which these fluctuations occur is much smaller than the one that characterizes the constrained profile. When $\Delta \theta_{1,2} \ll \theta \ll 1$, then $l_{1,2}-l_{0}+\theta x^{*} \approx x^{*} \Delta \theta_{1,2}$. 


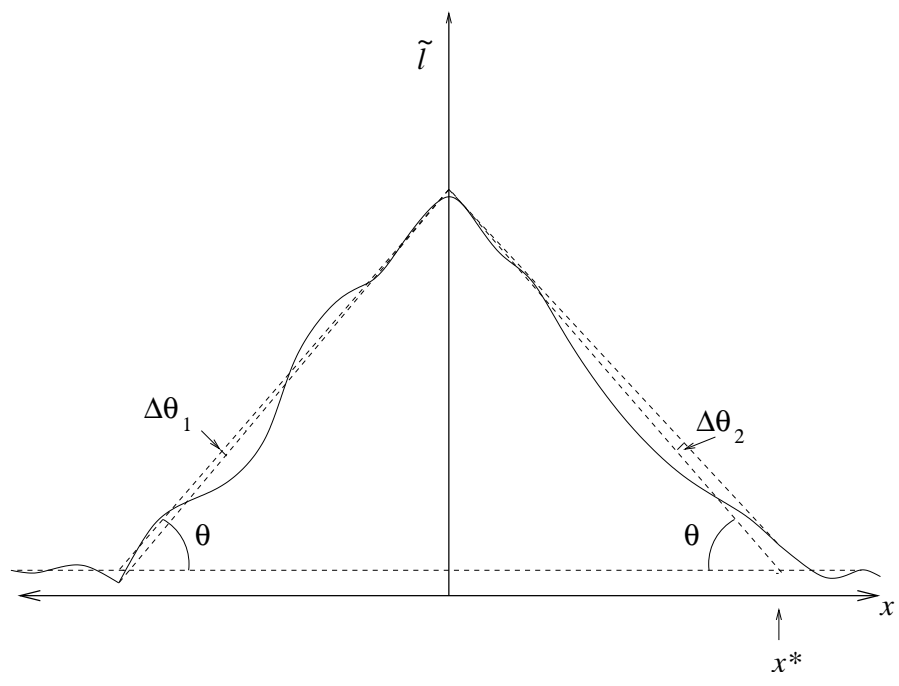

Figure A1. Plot of the typical relative interfacial position $\tilde{l} \equiv l-\alpha|x|$ close to the $2 \mathrm{D}$ wedge filling transition. $\Delta \theta_{1}$ and $\Delta \theta_{2}$ define the tilt fluctuations of the interface at $x^{*}$. See text for explanation.

Under these assumptions, the PDF for a wedge midpoint position $l_{0}$ and tilt angles $\Delta \theta_{1}$ and $\Delta \theta_{2}$ can be approximated as

$$
P\left(l_{0}, \Delta \theta_{1}, \Delta \theta_{2}\right) \approx 2 \Sigma(\theta-\alpha) e^{-2 \Sigma(\theta-\alpha) l_{0}} \frac{\Sigma x^{*}}{2 \pi} e^{-\frac{\Sigma x^{*}}{2}\left[\left(\Delta \theta_{1}\right)^{2}+\left(\Delta \theta_{2}\right)^{2}\right]}
$$

where the tilt angle ranges can be extended safely to the complete real axis. We define the tilt angles $\phi$ and $\phi^{\prime}$ as $\left(\Delta \theta_{1}+\Delta \theta_{2}\right) / 2$ and $\left(\Delta \theta_{1}-\Delta \theta_{2}\right) / 2$, respectively. Taking into account that in the scaling limit $x^{*} \approx l_{0} / \theta \approx l_{0} / \alpha$, Equation (A.3) can be expressed as:

$$
\begin{aligned}
P\left(l_{0}, \phi, \phi^{\prime}\right) & \approx \frac{2 \Sigma^{3 / 2} \sqrt{l_{0}}}{\sqrt{\pi \alpha}}(\theta-\alpha) e^{-\frac{\Sigma l_{0}}{\alpha}\left[2 \alpha(\theta-\alpha)+\phi^{2}\right]} \\
& \times \sqrt{\frac{\Sigma l_{0}}{\pi \alpha}} e^{-\frac{\Sigma l_{0}\left(\phi^{\prime}\right)^{2}}{\alpha}}
\end{aligned}
$$

Integrating all the values of $\phi^{\prime}$, we obtain:

$$
\begin{aligned}
& P\left(l_{0}, \phi\right) \approx \frac{2 \Sigma^{3 / 2} \sqrt{l_{0}}}{\sqrt{\pi \alpha}}(\theta-\alpha) e^{-\frac{\Sigma l_{0}}{\alpha}\left[2 \alpha(\theta-\alpha)+\phi^{2}\right]} \\
& \approx \frac{2 \Sigma^{3 / 2} \sqrt{l_{0}}}{\sqrt{\pi \alpha}}(\theta-\alpha) e^{-\frac{\Sigma l_{0}}{\alpha}\left(\theta^{2}-\alpha^{2}+\phi^{2}\right)}
\end{aligned}
$$

in agreement with Equation (81) and with the correct measure $D l_{0} \sim \sqrt{l_{0}} d l_{0}$.

\section{References}

[1] Gau H, Herminghaus S, Lenz P and Lipowsky R 1999 Science 28346

[2] Rascón C and Parry A O 2000 Nature 407986

[3] Bruschi L, Carlin A and Mistura G 2002 Phys. Rev. Lett. 89166101 
[4] Concus P and Finn R 1969 Proc. Natl. Acad. Sci. USA 63292

[5] Pomeau Y 1986 J. Colloid Interface Sci. 1135

[6] Hauge E H 1992 Phys. Rev. A 464994

[7] Rejmer K, Dietrich S and Napiórkowski M 1999 Phys. Rev. E 604027

[8] Parry A O, Rascón C and Wood A J 2000 Phys. Rev. Lett. 85345

[9] Parry A O, Wood A J and Rascón C 2001 J. Phys.: Condens. Matter 134591

[10] Parry A O, Rascón C and Wood A J 1999 Phys. Rev. Lett. 835535

[11] Parry A O, Wood A J and Rascón C 2000 J. Phys.: Condens. Matter 127671

[12] Parry A O, Greenall M J and Wood A J 2002 J. Phys.: Condens. Matter 141169

[13] Milchev A, Müller M, Binder K and Landau DP 2003 Phys. Rev. Lett. 90136101

[14] Milchev A, Müller M, Binder K and Landau DP 2003 Phys. Rev. E 68031601

[15] Forgacs G, Lipowsky R and Nieuwenhuizen Th M 1991 Phase Transition and Critical Phenomena vol 14, ed C Domb and J Lebowitz (New York: Academic)

[16] Lipowsky R 1985 J. Phys. A: Math. Gen. 18 L585

[17] Fisher M E 1986 J. Chem. Soc. Faraday Trans. 2821589

[18] Lipowsky R and Fisher M E 1986 Phys. Rev. Lett. 56472

[19] Lipowsky R and Fisher M E 1987 Phys. Rev. B 362126

[20] Abraham D B, Latrémolière F T and Upton P J 1993 Phys. Rev. Lett. 71404

[21] Indekeu J O and Robledo A 1993 Phys. Rev. E 474607

[22] Abraham D B, Parry A O and Wood A J 2002 Europhys. Lett. 60106

[23] Abraham D B and Maciolek A 2002 Phys. Rev. Lett. 89286101

[24] Albano E V, De Virgiliis A, Müller M and Binder K 2003 J. Phys.: Condens. Matter 15333

[25] Fisher M E and Jin A J 1992 Phys. Rev. Lett. 69792

[26] Jin A J and Fisher M E 1993 Phys. Rev. B 477365

[27] Nakanishi H and Fisher M E 1982 Phys. Rev. Lett. 491565

[28] Grinstein G and Ma S-K 1982 Phys. Rev. Lett. 49685

[29] Villain J 1982 J. Phys. (Paris) 43 L551

[30] Fisher D S 1986 Phys. Rev. Lett. 561964

[31] Huse D A, Henley C L and Fisher D S 1985 Phys. Rev. Lett. 552924

[32] Halpin-Healy T 1989 Phys. Rev. Lett. 62442

[33] Huse D A and Henley C L 1985 Phys. Rev. Lett. 542708

[34] Lipowsky R 1985 Phys. Rev. B 321731

[35] Lipowsky R and Fisher M E 1986 Phys. Rev. Lett. 56472

[36] Lipowsky R 1988 Europhys. Lett. 7, 255

[37] Bednorz A and Napiórkowski M 2000 J. Phys. A: Math. Gen. 33 L353

[38] Romero-Enrique J M, Parry A O and Greenall M J 2003 Preprint cond-mat/0311246 